\title{
Phosphocholine Steroid Conjugates: Are These Compounds the Mammalian Cardiotonic Steroids?
}

\author{
Fred Chasalow ${ }^{1,2, *}$ \\ ${ }^{1}$ Managing Partner, IOMA LLC \\ ${ }^{2}$ Visiting Professor, Department of Laboratory Medicine, VAMC , San Francisco, CA, USA \\ *Correspondence: Fred Chasalow: fchasalow@gmail.com
}

Received

\begin{abstract}
Cardiotonic glycoside toxins, originally isolated from plants or amphibians, have been proposed as mammalian cardiotonic hormones. This paper is a review and update of the discovery of [i] a new class of steroid hormones, [ii] the path for their biosynthesis and [iii] some preliminary data on their function. The compounds are phosphoester conjugates and share a characteristic structural feature, a lactone ring, with [a] one class of synthetic potassium sparing diuretics and with [b] plant and amphibian cardiotonic steroids. Purification was initially monitored by cross reaction with steroid sulfate assays and later with digoxin-specific assays. Six compounds were purified by HPLC to near homogeneity and characterized by Tandem mass spectroscopy (MS-MS) and ${ }^{31} \mathrm{P}-\mathrm{NMR}$. Three were digoxin-like materials (DLM) with 23 carbon atoms. The two extra carbon atoms form a spiral lactone E-ring. Several additional spiral lactones have been identified by MS-MS. In a pilot study, based on MS analysis, we evaluated phosphocholine steroid levels in individual serum samples in patients with pre-eclampsia $(n=20)$. The control group of normotensive pregnant women $(n=20)$ was used to estimate the mean and standard deviation. Twelve of the women with pre-eclampsia had a z-score over 2 for at least one of the four phosphocholine steroids. In contrast, only 1 sample from the normotensive women had a z-score over 2. The observation that there are two patterns, one with elevated phosphocholine steroid levels and one without the elevation, suggests that there may be two different underlying causes of pre-eclampsia. We now need to extend the study to determine which pattern predicts progression to life-threatening hypertension in pregnant women. This pilot study illustrates that it is possible to evaluate individual endogenous cardiotonic hormones without relying on antibodies developed to plant or amphibian toxins.
\end{abstract}

Keywords: Ionotropin; steroid phosphocholine esters; cardiotonic steroids; pre-eclampsia; endogenous ouabain; DLM; PTSD

\section{Introduction}

In the $18^{\text {th }}$ century, Withering recognized that an extract of foxglove, digoxin, was useful therapy for dropsy, now described as congestive heart failure [1]. In 1955, Szent-Gyorgyi proposed that digoxin wasn't really a drug but was a substitute for an unknown hormone [2]. This recognition led to a 35-year search for the endogenous hormone based on its detection by cross reaction with digoxin-specific antibodies [3]. In 1991, Hamlyn, starting with 80 liters of plasma, led a team that isolated $13 \mu \mathrm{g}$ of a material that seemed to be ouabain [4]. This was the initial observation that suggested that the cardiotonic steroids were not toxins but actually were endogenous hormones. More than 200 papers have now been published reporting endogenous ouabain levels in human disease based on cross reaction with an antibody developed to a cardiotonic steroid, typically ouabain or digoxin [5]. Although ouabain was characterized as an endogenous hormone, neither precursors nor metabolites of the steroid component have ever been detected by any direct analytical method [6]. 


\subsection{Nomenclature}

In total, this paper describes more than 20 novel compounds. For all but one, there is no trivial, recognized name and no familiar structure for any of them. For ease, phosphocholine esters will be designated as Cxxx; phosphoethanolamine esters will be designated as Exxx; Pxxx will be used to designate compounds for which the steroid fragment was observed but the conjugate was not confirmed by mass spectroscopy. For each symbol, the $x x x$ will be the $\mathrm{m} / \mathrm{z}$ from the steroid fragment as observed on mass spectroscopy in the cation mode. In most cases, the mass for the chemical formula of the steroid will be $m / z=x x+17$ Da because the phosphoester fragment $(m / z=184 \mathrm{Da})$ includes all four of the phosphate oxygen atoms, including the $3 \beta$-hydroxyl. For carboxyl steroid fragments, the mass for the chemical formula is $\mathrm{m} / \mathrm{z}=\mathrm{xxx}+15 \mathrm{Da}$ as the carboxyl fragment must be protonated to generate a cation. Note that all molecules containing only carbon, hydrogen, and oxygen atoms must have chemical formulas with an 'even" mass. Both nitrogen and phosphorus generate 'odd' masses except when an even number of them are present in the molecule $(1+1=2)$.

When the classical steroids were discovered, laboratories used mobility on thin layer chromatography (TLC) to identify individual steroids. However, as not all TLC methods were equivalent, the same compound was designated differently in different laboratories. The use of the mass of the MS fragment as part of the common name eliminates this source of confusion. The proposed stereochemistry is based on the stereochemistry of known enzymes catalyzing similar reactions. If the proposed stereochemistry is not correct, there must be another enzyme which has not yet been described.

\subsection{What was different in our approach that lead to the discovery}

Rather than starting with cross reaction to an antibody to a cardiotonic steroid, our path to discovery was dependent on two physiological starting points. First, Dr. Chasalow was investigating serum from newborn infants with Smith-Lemli-Opitz syndrome (SLO) [7]. Dr. Chasalow had noted that patients with SLO syndrome were potassium wasting and, in the newborn period, were missing two, unknown, steroid-like materials that cross-reacted with various steroid-sulfate antibodies [8]. However, the compounds were not present in serum from 2-week old normal infants. Hence, it was not possible to collect enough serum for characterization of the compounds. Second, Dr. Bradlow had noted that fluids from human breast cysts could be characterized on the basis of their electrolyte compositions [9]. Type 1 fluids had high potassium and low sodium levels while Type 2 electrolytes had the reverse. Together, we speculated that, to account for the difference in electrolytes, Type 1 fluids would have a compound that caused potassium accumulation but that the compound was not present in patients with SLO syndrome. From co-operating physicians, Dr. Bradlow obtained samples of both types of breast fluid. Dr. Chasalow extracted them with the methods used with the blood from newborn infants. The Type 1 fluids were very rich in the compounds that the infants with SLO couldn't make [10]. This recognition provided a pathway to isolation that was distinctly different from methods suitable for isolation of digoxin or ouabain.

\section{Results}

\subsection{Discovery}

\subsection{Isolation of C313, C341, C339, \& C337}

Our first source for successful isolation was outdated plasma obtained from our local blood bank [11]. As we couldn't recognize the DLM by UV absorption, we switched to LC-MS to supplement the DLM assay. There were two fractions. Fraction A had UV absorption over $240 \mathrm{~nm}$, consistent with conjugated alkenes, but was not a DLM. Fraction B did not have UV absorption over $240 \mathrm{~nm}$ but had DLM. 
Fraction $A$ had a peak ion at $\mathrm{m} / \mathrm{z}=496 \mathrm{Da}$ which was attributed to $\mathrm{M}+1$. The key observation was the presence of $\mathrm{m} / \mathrm{z}=184 \mathrm{Da}$ as the base ion. Authentic phosphocholine lipids all showed the base ion at $\mathrm{m} / \mathrm{z}=184 \mathrm{Da}$. The difference between the base peak and the $\mathrm{H}+$ mass ion was attributed to the steroid fragment. Thus, for the compound in Fraction A with $\mathrm{m} / \mathrm{z}=496 \mathrm{Da}$, the unknown steroid fragment would have $\mathrm{m} / \mathrm{z}=313 \mathrm{Da}$ and a fragment at that mass was present in the MS. The symbol for this compound would be C313. When the mass of the 3-hydroxyl group (17 Da) was added, C313 would have $\mathrm{m} / \mathrm{z}=330 \mathrm{Da}$ for the intact steroid molecule.

Table 1: Analysis of possible compositions of a molecule with $\mathrm{m} / \mathrm{z}=330 \mathrm{Da}$ (C313)

\begin{tabular}{|c|c|c|c|c|c|c|}
\hline Line & \# of C & \# of O & C + O & Hreq & Hmax & Delta \\
\hline 1 & 21 & 2 & 284 & 46 & 44 & -1 \\
\hline 2 & 21 & 3 & 300 & 30 & 44 & 7 \\
\hline 3 & 21 & 4 & 316 & 14 & 44 & 15 \\
\hline 4 & 22 & 3 & 312 & 18 & 46 & 14 \\
\hline 5 & 23 & 3 & 324 & 6 & 48 & 21 \\
\hline
\end{tabular}

Table 1 shows a trial and error analysis of possible chemical formulas containing only carbon, oxygen and hydrogen atoms with $\mathrm{m} / \mathrm{z}=330 \mathrm{Da}$. " $\mathrm{C}+\mathrm{O}$ " is the sum of the contribution of the carbon and oxygen atoms to the mass of the steroid. Hreq is the number of hydrogen atoms to complete the molecule as observed by MS. Hmax is twice the number of carbon atoms plus two. Delta is the exact number of delta elements needed to provide the Hreq. The line in RED shows the only combination that can make a steroid-like molecule - $\mathrm{C}_{21} \mathrm{H}_{30} \mathrm{O}_{3}$-and it would have a Delta of $7-4$ for the steroid rings, 2 for the alkenes at $\Delta 5$ and $\Delta 7$ and the ketone at carbon 20.
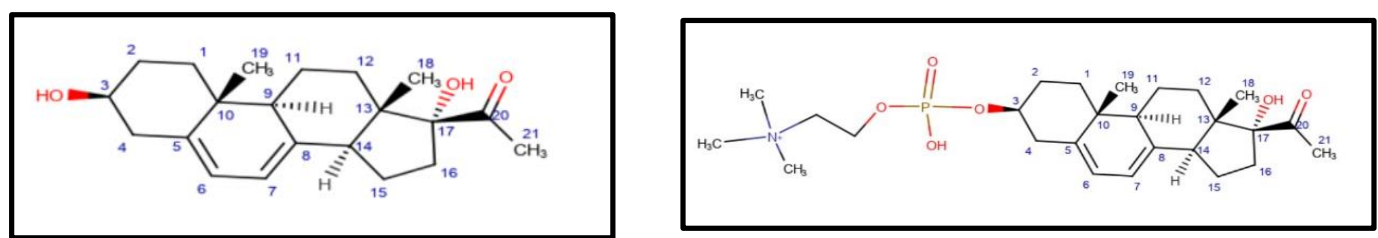

Figure 1. Left panel. Compound isolated by Shackleton from serum of a child with Smith-Lemli-Opitz syndrome [12]. Right Panel. Proposed structure of the H+ ion of C313 and which would have $\mathrm{m} / \mathrm{z}=496$ Da [11].

The major component of Fraction $B$ had an ion at $m / z=524$ Da. The steroid fragment had a mass of $\mathrm{m} / \mathrm{z}=341 \mathrm{Da}(524-183)$ and a symbol of C341. Table 2 shows the trial and error analysis for the composition of C341. Line 5 shows the only composition that fits the mass of $358 \mathrm{Da}$ is $\mathrm{C}_{23} \mathrm{H}_{34} \mathrm{O}_{3}$ and Delta must be 7 .

Table 2: Analysis of possible compositions of a molecule with $\mathrm{m} / \mathrm{z}=358 \mathrm{Da}$ (C341)

\begin{tabular}{|c|c|c|c|c|c|c|}
\hline Line & \# of C & \# of O & C + O & Hreq & Hmax & Delta \\
\hline 1 & 21 & 4 & 316 & 42 & 44 & 1 \\
\hline 2 & 21 & 5 & 332 & 26 & 44 & 9 \\
\hline 3 & 21 & 6 & 348 & 10 & 44 & 17 \\
\hline 4 & 22 & 3 & 312 & 46 & 36 & 0 \\
\hline 5 & 23 & 3 & 324 & 34 & 48 & 7 \\
\hline 6 & 23 & 4 & 340 & 18 & 48 & 15 \\
\hline 7 & 24 & 2 & 320 & 38 & 50 & 6 \\
\hline 8 & 24 & 3 & 336 & 22 & 50 & 14 \\
\hline
\end{tabular}

Trial and error analysis for the composition of a molecule with $\mathrm{m} / \mathrm{z}=358$ (C341). The line in RED shows the only combination that can make a steroid-like molecule. 

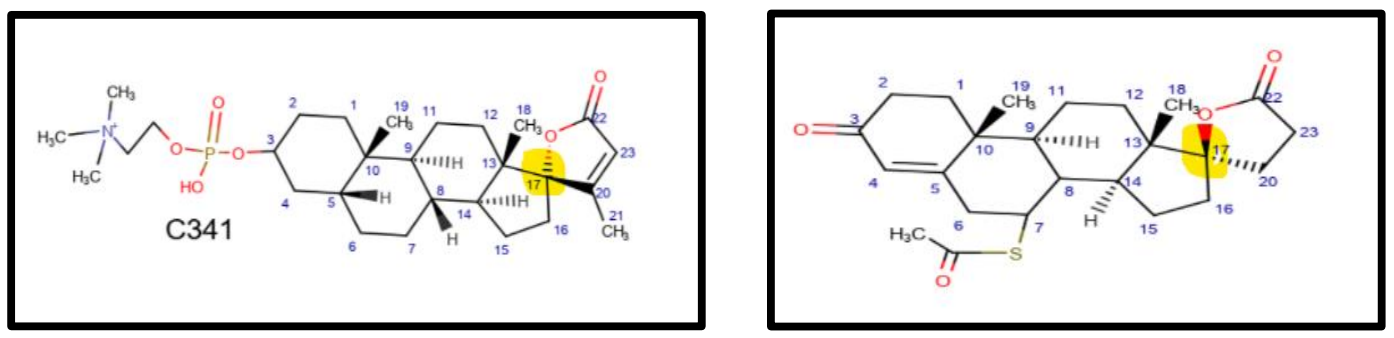

Figure 2. Structure of C 341 (left panel) and spironolactone (right panel).

Carbon 17 (highlighted in yellow) is a spiral carbon. Spiral carbons form a bridge between two rings. The proposed stereochemistry is based on well-known enzymes: $3 \beta$-hydroxy, $5 \beta$-reduction, and $17 \alpha$-hydroxylase. Note that direct reduction of $\Delta 4-3$ ketones leads to $5 \alpha$-stereochemistry. The numbering scheme is based on the standard numbering of steroids. In order to keep carbon 21 in its usual place, the extra carbons have been assigned numbers based on their possible origin in acyl coenzyme A.
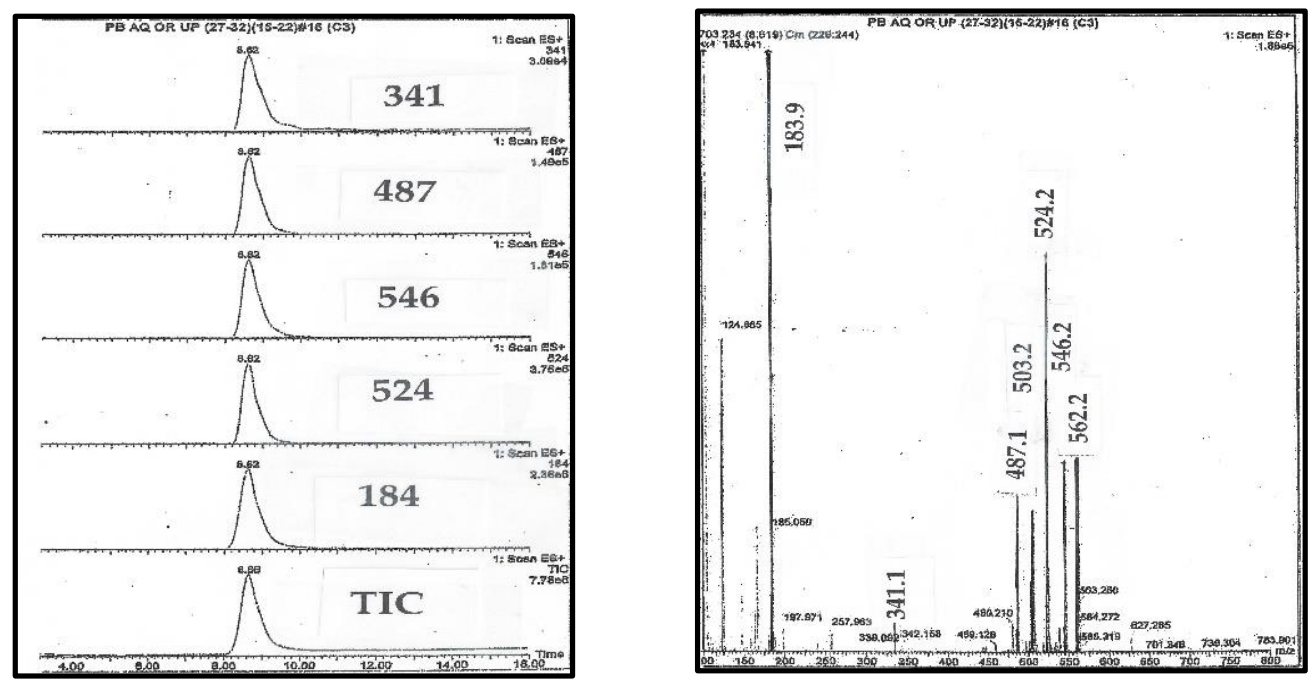

Figure 3 shows the LC-MS chromatogram obtained from C341 (Ionotropin) [11].

Left panel shows the LC chromatograms (Top to bottom). [i] $\mathrm{m} / \mathrm{z}=341 \mathrm{Da}$ - Ionotropin steroid fragment; [ii] $\mathrm{m} / \mathrm{z}=487 \mathrm{Da}-$ loss of trimethyl amine from Na+ salt; [iii] m/z=546 Da $-\mathrm{Na}+$ salt; [iv] m/z=524 Da $-\mathrm{H}+$ ion; [v] m/z=184 $\mathrm{Da}=$ phosphocholine fragment; and [vi] total ion count.

The right panel shows the MS obtained at the peak of the m/z=524 Da. Assignments: m/z=341 Da- steroid fragment; $\mathrm{m} / \mathrm{z}=562 \mathrm{Da}-\mathrm{K}+$ ion; $\mathrm{m} / \mathrm{z}=503 \mathrm{Da}$ loss of trimethylamine from the $\mathrm{K}+$ ion; $\mathrm{m} / \mathrm{z}=546 \mathrm{Da}-\mathrm{Na}+$ ion; $\mathrm{m} / \mathrm{z}=487$ $\mathrm{Da}$ - loss of trimethylamine from the $\mathrm{Na}+$ ion; $\mathrm{m} / \mathrm{z}=184 \mathrm{Da}$ - phosphocholine fragment; $\mathrm{m} / \mathrm{z}=124 \mathrm{Da}$ - possibly loss of trimethylamine from phosphocholine fragment.

Based on (a) the symmetrical peak shape and (b) the absence of ions at other retention times, the isolated material seemed to be essentially homogenous. In contrast to C313, C341 did not have a UV absorption band over $240 \mathrm{~nm}$, indicating it did not have a conjugated double bond.

In addition to C341, fraction $B$ had two less intense ions, one at $m / z=520 \mathrm{Da}(\mathrm{C} 337)$ and the other at $\mathrm{m} / \mathrm{z}=522 \mathrm{Da}$ (C339). C337 had a UV band over $240 \mathrm{~nm}$, indicating the presence of a conjugated double bond. The corresponding compositions from trial and error analysis are $\mathrm{C}_{23} \mathrm{H}_{30} \mathrm{O}_{3}$ and $\mathrm{C}_{23} \mathrm{H}_{32} \mathrm{O}_{3}$, respectively. Table 3 summarizes the MS fragmentation pattern of steroid phosphoesters. All of these compounds show similar fragmentation pattern, indicating that all are phosphocholine steroid esters. These three compounds plus C313 are the first description of endogenous steroid phosphoesters [11]. 
Table 3 Summary of mass ions and assignments for the phosphocholine esters with 23 carbon atoms and for DHEA-PC, a synthetic model compound

\begin{tabular}{|c|c|c|c|c|c|c|}
\hline Line & $\mathrm{m} / \mathrm{z}(\mathrm{Da})$ & Cation & $\mathrm{C} 337(\mathrm{Da})$ & $\mathrm{C} 339(\mathrm{Da})$ & $\mathrm{C} 341(\mathrm{Da})$ & DHEA-PC \\
\hline 1 & $\mathrm{M}+1$ & Hydrogen ion (Cxxx) & 520 & 522 & 524 & 454 \\
\hline 2 & $\mathrm{M}+23$ & Sodium ion & 542 & 544 & 546 & 476 \\
\hline 3 & $\mathrm{M}+23-59$ & Loss of trimethylamine & 483 & 485 & 487 & 417 \\
\hline 4 & $\mathrm{M}+39$ & Potassium ion & 558 & 560 & 562 & 492 \\
\hline 5 & $\mathrm{M}-182$ & Loss of phosphocholine & 337 & 339 & 341 & 271 \\
\hline 6 & $\mathrm{M}-182+17$ & Steroid formula mass & 354 & 356 & 358 & 288 \\
\hline 7 & 184 & Phosphocholine fragment & 184 & 184 & 184 & 184 \\
\hline
\end{tabular}

$\mathrm{C} 337\left(\mathrm{C}_{23} \mathrm{H}_{30} \mathrm{O}_{3}\right), \mathrm{C} 339\left(\mathrm{C}_{23} \mathrm{H}_{32} \mathrm{O}_{3}\right)$, and $\mathrm{C} 341\left(\mathrm{C}_{23} \mathrm{H}_{34} \mathrm{O}_{3}\right)$ were first isolated from Fraction B from human plasma. Later, multi-mg samples were isolated from porcine and bovine blood, which had been obtained at local slaughterhouses. Each of these compounds was also isolated to $>90 \%$ homogeneity from bovine adrenal extracts. DHEA-PC was synthesized in the laboratory [13]. The ions on line 3 have odd masses, suggesting the loss of trimethyl amine fragment from the phosphocholine steroid.

\subsubsection{NMR analysis of steroids with 23 carbon atoms.}

${ }^{1} \mathrm{H}-\mathrm{NMR}$ analysis is shown in Figure 4. The spectra are complex with many different types of splitting patterns, which is typical of steroids. Two features were identifiable. First, there were no long chain alkyl groups similar to a fatty acid or fatty alcohol. Second, there were three "unsplit" methyl groups as shown in the inset. Two of these could be assigned to the angular methyl groups at carbon 18 and carbon 19. The third one is consistent with the methyl group at carbon 21, which would be also be unsplit as the adjacent carbon could be either a ketone or an alkene.

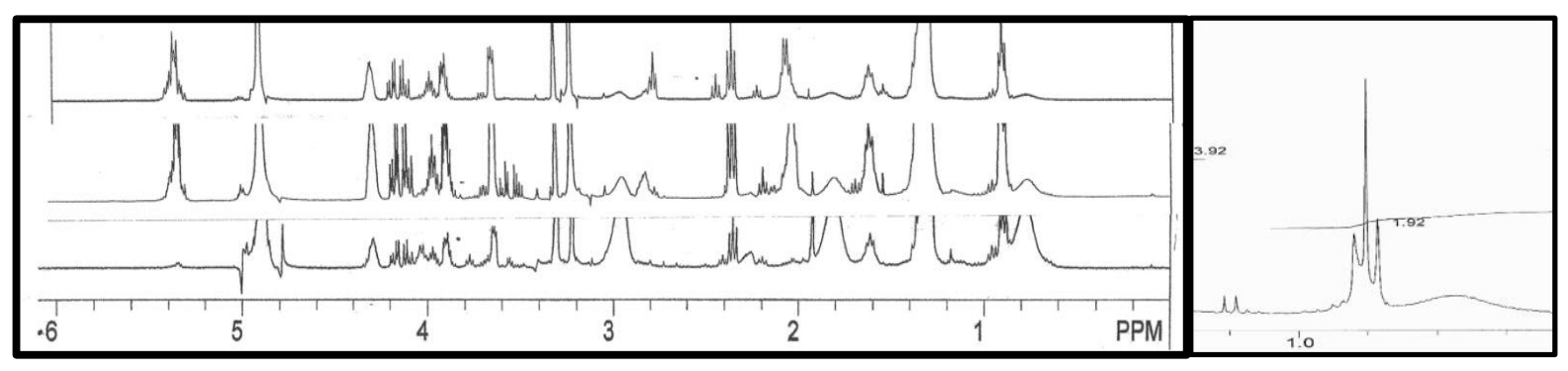

Figure 4. H-NMR analysis of spiral lactones with 23 carbon atoms Top: C337; Middle: C339; Bottom: C341 [11].

${ }^{13} \mathrm{C}-\mathrm{NMR}$ analysis provided a limited amount of new data. It was not consistent with a long chain alkyl group, thus eliminating structures similar to platelet activating factor and other phospholipids.

Analysis of the ${ }^{31 P-N M R, ~ s h o w n ~ i n ~ F i g u r e ~ 5, ~ c o n f i r m s ~ t h e ~ m o l e c u l e ~ h a s ~ o n e ~ p h o s p h o r u s ~ a t o m . ~}{ }^{31 P-}$ NMR chemical shifts vary up to several hundred, depending on the oxidation state of the phosphorus atom, confirming that these two molecules have phosphorus in a similar oxidation state.

If all of the other atoms were carbon, hydrogen, or oxygen, the mass would be odd. This indicates that there must be a nitrogen atom in the molecule because nitrogen is the only common atom that would also generate an odd molecular mass, and two odds make it even. Prior to these investigations, there were no known endogenous steroid phosphoesters. DHEA-3-phosphocholine ester was synthesized in the laboratory and used as a model compound [13]. 

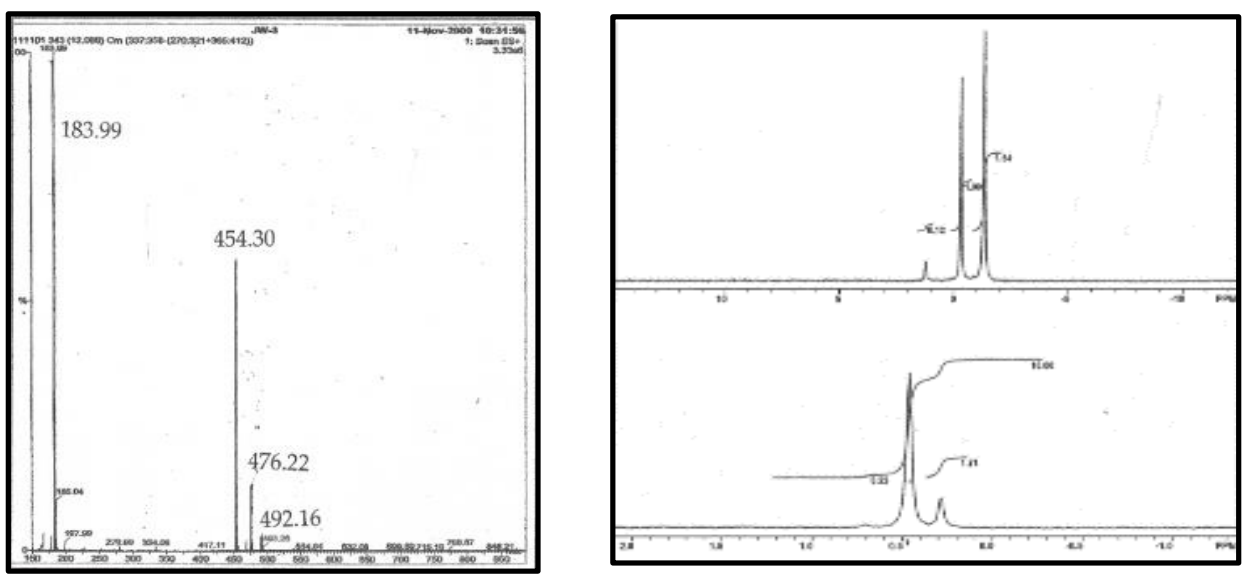

Figure 5. Confirmation of phosphocholine esters by ${ }^{31} \mathrm{P}-\mathrm{NMR}$ (11) Left panel MS of synthetic DHEA-PC Right Panel Top: ${ }^{31 P-N M R ~ o f ~ D H E A-P C ; ~ B o t t o m ~: ~}{ }^{31 P-N M R ~ o f ~ C 341 . ~[11] ~}$

\subsubsection{Biosynthesis of steroids with 23 carbon atoms -part 1}

There are two ways to generate a steroid with 23 carbon atoms: either by [a] adding two carbon atoms to a 21-carbon atom precursor or by [b] removing four carbon atoms from a sterol, perhaps cholesterol. Starting with side chain labeled cholesterol, Burstein could not find any evidence for a four carbon atom fragment [14] and we have not observed any ion consistent with cholesterol phosphocholine ester in any LC-MS chromatogram. Further, two of the isolated compounds, C313 and C337, have UV absorption characteristic of conjugated double bonds. As cholesterol does not have a similar UV absorption band, it can't be a precursor of C313. This suggestion is confirmed by the lack of reports of the use of cholesterol tracer to isolate a DLM, as has been done for the other steroid hormones. That dog didn't bark in the night. Slominski showed that the side chain cleavage enzyme cleaved 7-dehydrocholesterol to 7dehydropregnenolone [15]. His team also isolated its 17 $\alpha$-hydroxy metabolite [16], which we would designate P313, if it were phosphorylated. C337 and C339 would fit in the biosynthesis pathway between C313 and C341. The proposed pathway is shown in Scheme 1. The enzyme in the gap has not been described.
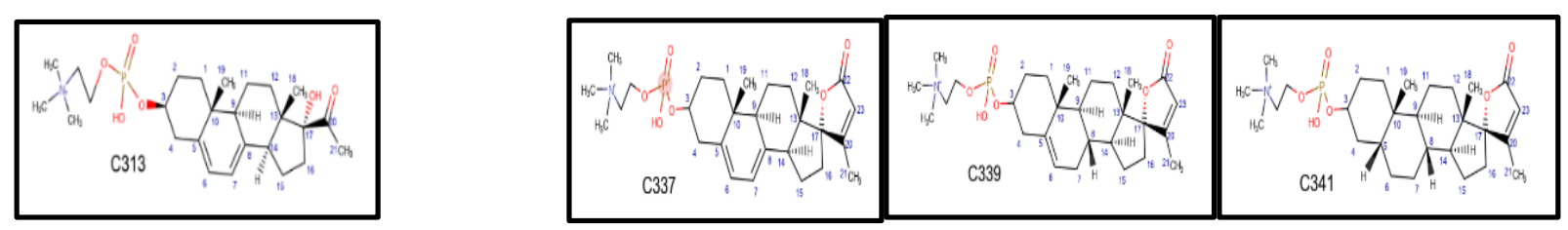

Scheme 1. These four panels show the structures proposed for the first four steroid phosphoesters that we isolated. The conversion of C337 to C339 is catalyzed by 7-dehydrostrol reductase [17]. The conversion of C339 to C341 is catalyzed by 5-sterol reductase [18]. However, the conversion of C313 to C337 has not been described - the gap.

\subsubsection{The Structure of C341}

Figure 2 compares the proposed structure of C341 to that of spironolactone. The common feature of these compounds is the lactone E-ring. Table 4 summarizes the different observations that support the proposed structure for C341 (Ionotropin). 
Table 4: Summary of the observations about the structure of Ionotropin (C341):

- Hydrogen ion form has $\mathrm{m} / \mathrm{z}=524 \mathrm{Da}$

- Loss of the trimethylamine (59 Da) from the $\mathrm{Na}+$ ion $(\mathrm{m} / \mathrm{z}-=546 \mathrm{Da})$ confirms the presence of a choline

- $\quad{ }^{31} \mathrm{P}-\mathrm{NMR}$ confirms the presence of phosphate

- Fragment $\mathrm{m} / \mathrm{z}=184$ Da indicates a phosphocholine

- The composition of the 341 Da fragment must be $\mathrm{C}_{23} \mathrm{H}_{34} \mathrm{O}_{3}$ with $\Delta=7$. No other combination of carbon, hydrogen and oxygen atoms makes a molecule. See Table 2. Isomers are not prohibited.

- $\quad$ C339 and C337 differ from C341 by 1 and 2 alkene groups, respectively, as illustrated in Scheme 1

- $\quad$ C341 cross-reacts with digoxin specific antibodies and is a DLM by definition, whether or not every structural element has been correctly identified.

- There is no evidence that C341 is a glycoside rather than a phosphocholine ester.

- $\quad$ Patients with 21-hydroxylase have C341 in their serum, confirming carbon 21 is a methyl group [11].

\subsubsection{Isolation and characterization of C361 and C363}

Frozen bovine adrenals were obtained from Pel Freeze Biologicals (Rogers, AR). Tissue was ground and extracted as described in Methods. In addition to the four compounds isolated from human plasma, there were several other phosphoesters present. C361 (m/z=544 Da) and C363 (m/z=546 Da) were present in sufficient concentration to be purified to greater than $90 \%$ homogeneity [19]. Table 5 shows the trial and error analysis of the chemical composition of C363.

Table 5: Analysis of possible compositions of C363

If formula mass is $380 \mathrm{Da}$

\begin{tabular}{||c|c|c|c|c|c|c||}
\hline Line & $\begin{array}{c}\text { \# of } \\
\text { C }\end{array}$ & $\begin{array}{c}\text { \# of } \\
\text { O }\end{array}$ & $\begin{array}{c}\text { C \& } \\
\text { O }\end{array}$ & Hreq & Hmax & $\Delta$ \\
\hline 1 & 21 & 5 & 332 & 46 & 44 & -1 \\
\hline 2 & 21 & 6 & 348 & 30 & 44 & 7 \\
\hline 3 & 22 & 5 & 344 & 34 & 46 & 6 \\
\hline 4 & 23 & 3 & 324 & 54 & 48 & 3 \\
\hline 5 & 23 & 4 & 340 & 38 & 48 & 5 \\
\hline 6 & 23 & 5 & 356 & 22 & 48 & 13 \\
\hline 7 & 24 & 3 & 336 & 38 & 50 & 6 \\
\hline 8 & 24 & 4 & 352 & 26 & 50 & 14 \\
\hline
\end{tabular}

\begin{tabular}{|c|c|c|c|c|c|c|}
\hline Line & $\begin{array}{c}\text { \# of } \\
\text { C }\end{array}$ & $\begin{array}{c}\text { \# of } \\
\text { O }\end{array}$ & $\begin{array}{c}\text { C \& } \\
\text { O }\end{array}$ & Hreq & Hmax & $\Delta$ \\
\hline 11 & 21 & 5 & 332 & 48 & 44 & -2 \\
\hline 12 & 21 & 6 & 348 & 32 & 44 & 6 \\
\hline 13 & 22 & 5 & 344 & 36 & 46 & 5 \\
\hline 14 & 23 & 3 & 324 & 56 & 48 & -4 \\
\hline 15 & 23 & 4 & 340 & 40 & 48 & 4 \\
\hline 16 & 23 & 5 & 356 & 24 & 48 & 12 \\
\hline 17 & 24 & 3 & 336 & 40 & 50 & 5 \\
\hline 18 & 24 & 4 & 352 & 28 & 50 & 13 \\
\hline
\end{tabular}

The parent ion has $m / z=546 \mathrm{Da}$ and we observed a fragment at $\mathrm{m} / \mathrm{z}=363 \mathrm{Da}$ just as we expected.

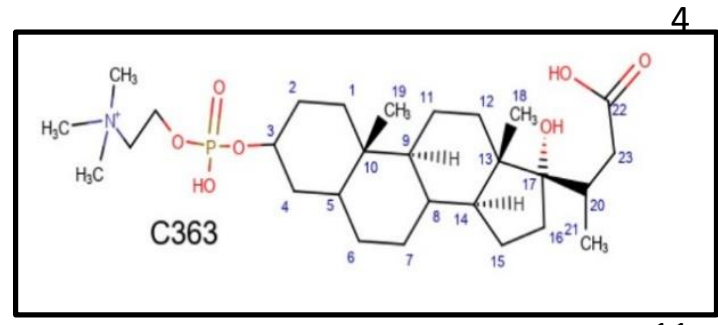

Figure 6. $\mathrm{C} 363$ with $\mathrm{m} / \mathrm{z}=546 \mathrm{Da}$.

To be detected in positive mode, the ion must be protonated. Thus, the chemical formula is consistent with mass of $378 \mathrm{Da}(\mathrm{Cxxx}+15)$.
Lines 11-18 show the analysis of $\mathrm{m} / \mathrm{z}=380 \mathrm{Da}$. However, none of these lines identify an acceptable composition. If the E-ring hasn't closed, then C363 would be a carboxylic acid and an anion. To get a positive fragment, we have to add two $\mathrm{H}$ atoms. Thus, for carboxylic phosphoesters, the actual $\mathrm{m} / \mathrm{z}$ value is only 15 Da above the observed steroid mass fragment, rather than $17 \mathrm{Da}$, as it is for the spiral lactones. Lines 1-8 show the analysis of $\mathrm{m} / \mathrm{z}=378 \mathrm{Da}$ and Line 4 indicates a composition of $\mathrm{C}_{23} \mathrm{H}_{38} \mathrm{O}_{4}$.

The mass difference of 22 Da between C363 and C341 requires 3 significant differences in structure: [i] 
C363 is a carboxylic acid while C341 is a spiral lactone, which adds 18 Da, [ii] ] the C20-C23 alkene in C341 must be reduced in C363, which adds 2 Da and [iii] the carboxylic acid of C363 must be protonated, which adds 2 Da more to its mass to get to C363. Figure 7 shows 3D representation of isomers of carboxylic acids C357 and C359. The actual pair of interest is C361 and C363, but the figures were prepared with C357 and C359 because the double bonds improve visualization of the structures. When the C20-C23 alkene bond is reduced, as in C363, the two oxygen atoms (shown in red) can't get close enough to complete the E-ring lactone.
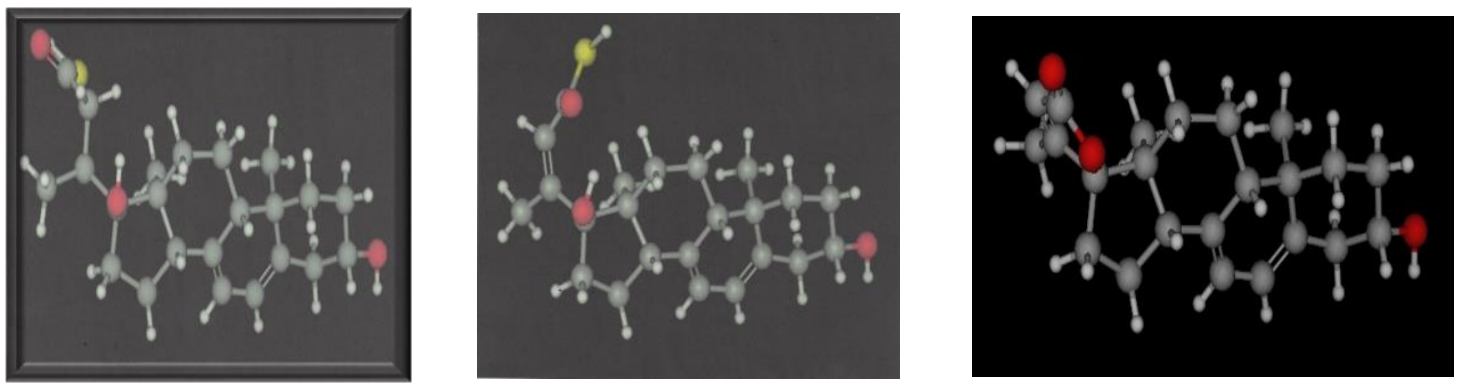

Figure 7 Left Panel - P359; Center Panel - P357; Right Panel - P337.

Colors of the atoms: Red - oxygen; Yellow- sulfur of coenzyme A; Grey - carbon; White - hydrogen. This is a 3D representation of the structures of the carboxylic acids. In this representation, Carbon 3 is on the right, the reverse of the usual presentation. The phospho-ester is attached at the oxygen on Carbon 3.

These three panels show the consequence of reduction of the C20-C23 double bond. When the C20-C23 double bond is reduced, as in the left-hand panel, the lactone ring can't be formed because the two oxygen atoms are not in juxtaposition. Only if the C20-C23 alkene bond is intact, as shown in the middle panel, can the E-Ring be closed. The completed E-ring, P337, is shown in the right-hand panel.

This discussion is important for several reasons: first, it confirms the C20-C-23 alkene in the spiral lactones, and, second, it indicates that the carboxylic acids need not be on the biosynthesis pathway, depending on which alkene is present. Note that C363 and C341 have both alkene bonds ( $\Delta 5-6$ and $\Delta 7-8)$ reduced, as do both digoxin and ouabain. Thus, it is possible that C363 might fit into the binding site for the phosphocholine portion of the cardiotonic steroids and block their function.

\subsubsection{Phosphoethanolamines in bovine adrenals}

In addition to the phosphocholine esters, there was a group of compounds that did not have the. characteristic fragment at $\mathrm{m} / \mathrm{z}=184 \mathrm{Da}$ but shared the same steroid fragment ions. The parent ions were $\mathrm{m} / \mathrm{z}=42$ Da less than the parent ions for the corresponding Cxxx compounds. For example, there were two peaks for P313. One compound had $\mathrm{m} / \mathrm{z}=496 \mathrm{Da}$ and had the $\mathrm{m} / \mathrm{z}=184 \mathrm{Da}$ fragment characteristic of the phosphocholine compounds. The second compound had $\mathrm{m} / \mathrm{z}=454 \mathrm{Da}$ and did not show a fragment at $\mathrm{m} / \mathrm{z}=184 \mathrm{Da}$. The $\mathrm{m} / \mathrm{z}=42 \mathrm{Da}$ difference is consistent with a phosphoethanolamine ester and would be designated as E313. In brief, in the adrenal extracts, for each of the Pxxx compounds that we detected, there was both a Cxxx and an Exxx. However, Exxx compounds were only present at trace levels (or not at all) in serum extracts [19].

Choline is an essential nutrient. As a consequence, the choline kinase pathway is probably not functional in mammals. The basic pathway seems to be the Kennedy pathway - CDP-ethanolamine donation to a sterol and subsequent $\mathrm{N}$-methylation to generate the phosphocholine in situ [20]. There are two ways to generate CDP-ethanolamine. It can be generated either from ethanolamine kinase or from CDP-serine and decarboxylation. The latter pathway seems more likely because serine is not an essential amino acid [21]. In either pathway, the final step would be N-methylation. There are several ACTH- 
dependent adrenal enzymes that N-methylate amines [22]. Thus, the presence of the Exxx compounds suggests the adrenal is the site of synthesis for the corresponding Cxxx compounds. Perhaps, the Exxx are a storage form in a special organelle. In response to ACTH, the N-methylation would be stimulated and the Cxxx secreted. A similar process occurs with norepinephrine and epinephrine. However, the enzymes that catalyze these processes for the steroid phosphoesters have not been identified or described.

\subsubsection{Phosphoesters in eggs}

Unfertilized, fresh eggs were obtained from a local farmer. The eggs were collected on Friday, purchased on Saturday and extracted on Monday. Each egg was 'floated' (Fresh eggs don't float.) to confirm their freshness [23]. Neither extracts from the whites nor the yolks contained Exxx steroids, indicating biosynthesis probably doesn't occur after the shell is formed. DLM was present in egg white extracts but not in egg yolk extracts. MS-MS confirmed that the major Cxxx extracts in the yolk extracts was C313, which is not a DLM. The egg white extracts contained C337, C339 and C361. These were present as both the $\mathrm{Na}+$ cation and the $\mathrm{K}+$ cation. C337 and C339 are steroid spiral lactones and are DLM. C361 is a carboxylic acid. This study shows the steroid phosphoesters are not limited to mammals but are also present in avian species [24].

In preparing this review, I also reviewed the original extracts from turkey and chicken serum. Ions corresponding to $\mathrm{C} 337$ and $\mathrm{C} 339$, as both the $\mathrm{Na}+$ and $\mathrm{K}+$ ions, were present, but there was no $\mathrm{C} 341$ in any of the samples. As there are three alkenes in C337, (C5-C6, C7-C8, \& C20-C23) one of the alkenes was not reduced. If C20-C23 was reduced, then the lactone ring couldn't be formed. See Figure 7 for the 3D drawings showing the consequence of the reduction of $\Delta 20-23$ alkene. In humans, C313 is accumulated in serum from patients with SLO, indicating that the $\Delta 5-6$ reductase doesn't reduce $\Delta 7-8$ sterols. Thus, if the avian enzymes have similar substrate specificity, then the C5-C6 alkene must still be intact. An example of a somewhat similar circumstance is testosterone and $5 \alpha$-dihydrotestosterone. I don't have any specific insight as to the biological significance of this observation in the birds.

\subsubsection{Phosphocholine esters in bovine ovarian extracts:}

Frozen bovine ovaries were obtained from Pel Freeze Biologicals (Rogers, AR). Tissue was ground, extracted as described in Methods, and analyzed by MS-MS. Mass ions characteristic of C313, C337, C339, and C341 were present in the extracts. These were noted but not further investigated. However, there were two additional compounds identified at m/z $554 \mathrm{Da}$ (C371) and m/z=510 Da (E369).

\begin{tabular}{|c|c|c|c|c|c|c|c|c|c|c|c|c|c|}
\hline Line & $\begin{array}{l}\text { \# } \\
\text { of } \\
\text { C }\end{array}$ & $\begin{array}{l}\# \\
\text { of } \\
\mathrm{O}\end{array}$ & $\mathrm{C} \& \mathrm{O}$ & Hreq & Hmax & Delta & Line & $\begin{array}{l}\# \\
\text { of } \\
C\end{array}$ & $\begin{array}{l}\# \\
\text { of } \\
\mathrm{O}\end{array}$ & $\mathrm{C} \& \mathrm{O}$ & Hreq & Hmax & Delta \\
\hline 1 & 23 & 4 & 340 & 48 & 48 & 0 & 11 & 23 & 4 & 340 & 46 & 48 & 1 \\
\hline 2 & 23 & 5 & 356 & 32 & 48 & 8 & 12 & 23 & 5 & 356 & 30 & 48 & 9 \\
\hline 3 & 24 & 3 & 336 & 52 & 50 & -1 & 13 & 24 & 3 & 336 & 50 & 50 & -0 \\
\hline 4 & 24 & 4 & 352 & 36 & 50 & 7 & 14 & 24 & 4 & 352 & 34 & 50 & 8 \\
\hline 5 & 24 & 5 & 368 & 20 & 50 & 15 & 15 & 24 & 5 & 368 & 18 & 50 & 16 \\
\hline 6 & 25 & 3 & 352 & 40 & 52 & 6 & 16 & 25 & 3 & 352 & 38 & 52 & 7 \\
\hline 7 & 25 & 4 & 364 & 24 & 52 & 14 & 17 & 25 & 4 & 364 & 22 & 52 & 15 \\
\hline
\end{tabular}

Table 6. Trial and error analysis of possible chemical formulas for C371 and E369

Left Panel: C371. Parent ion at $\mathrm{m} / \mathrm{z}=554 \mathrm{Da}$ Based on $\mathrm{m} / \mathrm{z}=388 \mathrm{Da}$
Right Panel: E369. Parent ion at m/z-510 Da Based on $\mathrm{m} / \mathrm{z}=386 \mathrm{Da}$ 

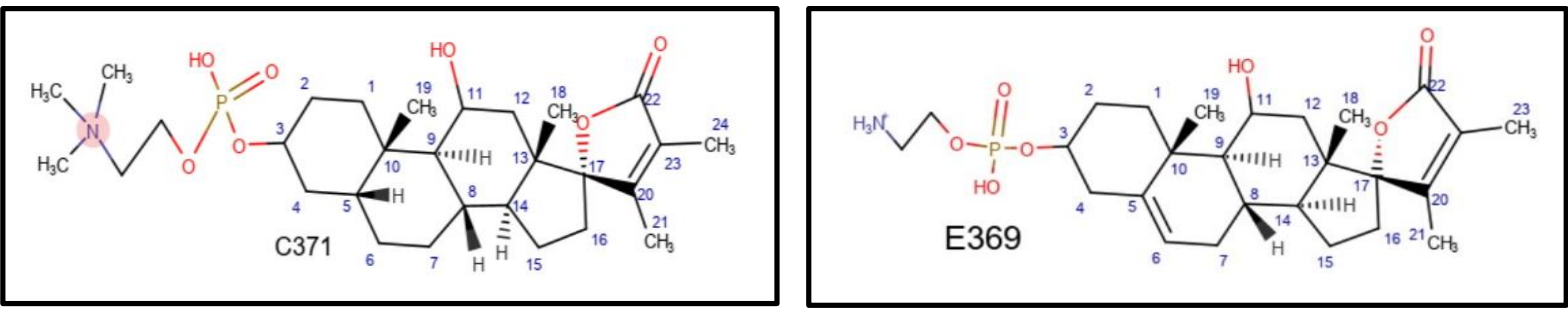

94

95

96

97

98

99

100

101

102

103

104

105

106

107

108

109

110

111

112

113

114

115

116

117

118

119

120

121

122

123

124

125

Figure 8. Structures for C371 and E369
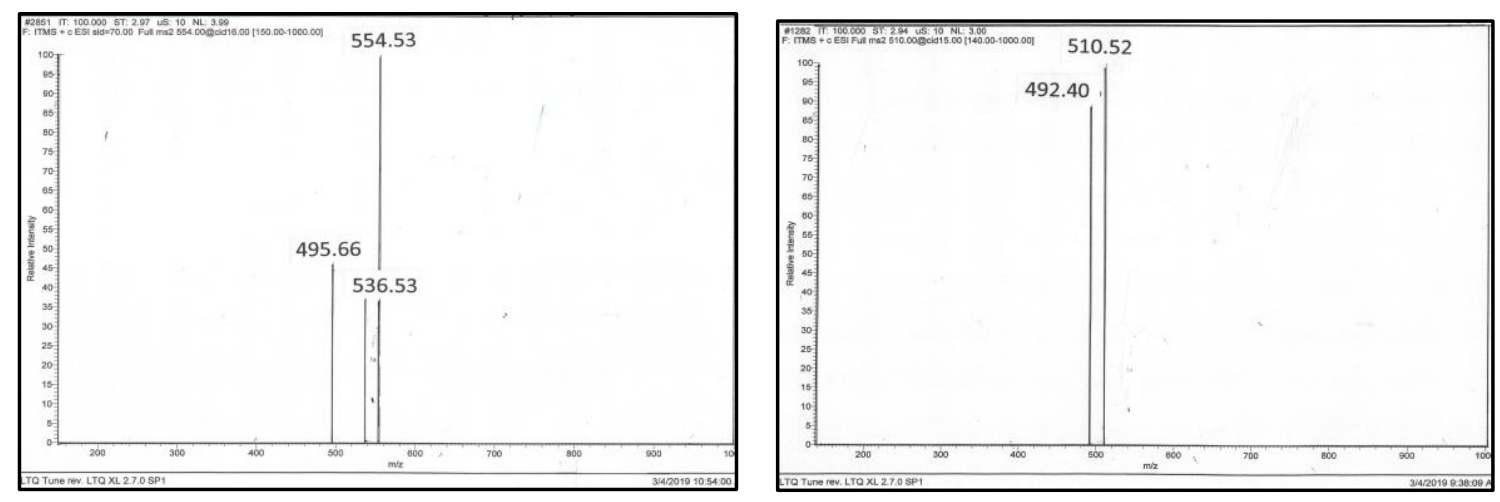

Figure 9. MS-MS data obtained from the extract of bovine ovary. Left panel: MS-MS analysis of ion $\mathrm{m} / \mathrm{z}=554 \mathrm{Da}$.

Right panel: MS-MS analysis of $\mathrm{m} / \mathrm{z}=510 \mathrm{Da}$.

The mass of the fragments derived from the ion at $m / z=554$ Da were $m / z=495$ Da and $m / z=536$ Da (Figure 9). These correspond to loss of $59 \mathrm{Da}$ and loss of $18 \mathrm{Da}$, respectively. The loss of $59 \mathrm{Da}$ indicates loss of trimethylamine from the phosphocholine ester. Thus, the symbol for this compound would be C371 based on $\mathrm{m} / \mathrm{z}=554 \mathrm{Da}-183 \mathrm{Da}$. Loss of an $18 \mathrm{Da}$ fragment is typical of a hydroxyl group. This is the first fragmentation pattern we observed consistent with a hydroxylated steroid. Table 6 suggests that the composition of the steroid is $\mathrm{C}_{24} \mathrm{H}_{36} \mathrm{O}_{4}$ as shown in red on line 4.

The mass of the fragment derived from the ion at $\mathrm{m} / \mathrm{z}=510$ Da was at $\mathrm{m} / \mathrm{z}=492$ which would be generated by loss of water from a hydroxyl group. There was no ion corresponding to loss of trimethylamine. The steroid fragment would have $\mathrm{m} / \mathrm{z}=369 \mathrm{Da}(510-161=369 \mathrm{Da})$. The corresponding symbol would be E369. Line 14 of Table 6 suggests its composition is - $\mathrm{C}_{24} \mathrm{H}_{34} \mathrm{O}_{4}$-indicating a $\Delta 5-6$ alkene would be present, similar to that of C339. In the bovine adrenal extracts, there was an unexplained steroid fragment at $\mathrm{m} / \mathrm{z}=389 \mathrm{Da}$ and a parent ion at $\mathrm{m} / \mathrm{z}=530 \mathrm{Da}$. This is consistent with E389 which would be the carboxylic acid precursor to E369. Together, the observations suggest a steroid fragment with 24 carbon atoms. This is the first description of a steroid with 24 carbon atoms.

There are four common steroid hydroxylase enzymes: $11 \beta$-hydroxylase, $17 \alpha$-hydroxylase, 18hydroxylase and 21-hydroxylase. In order to generate a lactone ring, a steroid lactone must have a $17 \alpha$ hydroxyl group. Both 18-hydroxylase and 21-hydroxylase act on the corresponding axial methyl groups, which we know from the NMR data, are intact, and neither can fragment with a loss of 18 Da. Hamlyn investigated biosynthesis of DLM in bovine adrenal cortex cells in culture. Metyrapone, an 11-hydroxylase inhibitor, decreased synthesis of endogenous ouabain [25]. Thus, both observations suggest carbon 11 for the extra hydroxyl group, probably with the $\beta$-stereochemistry, just like glucocorticoids.

Table 7 summarizes the biosynthetic path based on the compounds we have detected by MS-MS analysis. Note the similarity to the compounds with 23 carbon atoms that we purified to homogeneity. 
Table 7: Steps in the pathway for biosynthesis of C371 from E313

$\begin{array}{lcll}\text { Step } & \text { Symbol } & \text { Product } & \mathrm{m} / \mathrm{z}(\mathrm{H}+) \\ 1 & \text { E313 } & \text { E329 } & 470 \mathrm{Da} \\ 2 & & & \\ 3 & & \text { E389 } & 530 \mathrm{Da} \\ 4 & \text { E389 } & \text { E369 } & 510 \mathrm{Da} \\ 5 & \text { E369 } & & 512 \mathrm{Da} \\ 6 & & \text { C371 } & 554 \mathrm{Da}\end{array}$

Enzyme

11ß-hydroxylase

$\Delta 7$ sterol reductase

Condensation

Formation of spiral lactone

$\Delta 5 \beta$-sterol reductase

N-Methylation

Symbols and masses in red have been identified by MS. The open spots indicate that the intermediate compounds have not been detected. The order of the steps without identified intermediates has not been confirmed.

\subsubsection{Biosynthesis of steroids with 23 (or more) carbon atoms -part 2}

In the original paper describing the steroids with 23 carbon atoms, we proposed a malonic acid condensation driven by decarboxylation [11]. Now that we know about the compounds with 24 carbon atoms and possibly 25 carbon atoms, that process is no longer tenable. Scheme 2 shows the proposed role of acyl coezyme A as the origin of the additional carbon atoms.

Table 8 Phosphocholine steroid esters detected by MS

\begin{tabular}{|c|c|c|c|c|c|c|c|}
\hline $\mathbf{m} / \mathbf{z}$ & Symbol & \# & Serum & Tissue & Extract & & Other structural features \\
\hline (Da) & & of $\mathrm{C}$ & & Adrenal & Ovary & Alkenes & \\
\hline 496 & C313* & 21 & $\mathrm{AC}$ & $\mathrm{AB}$ & & $\Delta 5-\Delta 7$ & \\
\hline 512 & C329 & 21 & A & & & $\Delta 5-\Delta 7$ & 11ß-hydroxyl-C313 \\
\hline 520 & C $337^{*}$ & 23 & A & $\mathrm{AB}$ & & $\Delta 5-\Delta 7-\Delta 20$ & Lactone \\
\hline 522 & $\mathrm{C} 339^{*}$ & 23 & A & $\mathrm{AB}$ & & $\Delta 5-\Delta 20$ & Lactone \\
\hline 524 & $\mathrm{C} 341^{*}$ & 23 & $\mathrm{AC}$ & $\mathrm{AB}$ & & $\Delta 20$ & Lactone \\
\hline 544 & $\mathrm{C} 361^{*}$ & 23 & A & $\mathrm{AB}$ & & $\Delta 20$ & Carboxylic acid \\
\hline 546 & C $363^{*}$ & 23 & A & B & & none & Carboxylic acid \\
\hline $\mathrm{D}$ & C353 & 24 & $\mathrm{AC}$ & & & $\Delta 5-\Delta 20$ & Lactone - 23 methyl-C339 \\
\hline $\mathrm{D}$ & C355 & 24 & $\mathrm{AC}$ & & & $\Delta 20$ & Lactone - 23-methyl-C341 \\
\hline 552 & C369 & 24 & $\mathrm{AC}$ & & $\mathrm{AB}$ & $\Delta 5-\Delta 20$ & Lactone - 23 methyl - 11ß-hydroxyl-C339 \\
\hline 554 & C371 & 24 & $\mathrm{AC}$ & & A & $\Delta 20$ & Lactone - 23-methyl -11 $\beta$ - hydroxyl-C341 \\
\hline 572 & C389 & 24 & ND & $\mathrm{AB}$ & & $\Delta 5-\Delta 20$ & Carboxyl -23 methyl -11 $\beta$-hydroxyl-C339 \\
\hline $\mathrm{D}$ & P381 & 25 & C & $\mathrm{E}$ & & $\Delta 5 \Delta 20$ & Lactone -23 acetyl-C339 \\
\hline
\end{tabular}

*Purified to near homogeneity

$\mathrm{m} / \mathrm{z}$ is the observed mass ion of the $\mathrm{H}+$ mass ion. The mass of the $\mathrm{Na}+$ ion would $22 \mathrm{Da}$ higher.

$\mathrm{A}=$ Phosphocholine ion detected as either the $\mathrm{H}+$ ion or $\mathrm{Na}+$ ion

$\mathrm{B}=$ Phosphoethanolamine ion detected in mass spectrum

$\mathrm{C}=$ Steroid fragment detected, but conjugate not detected $-\mathrm{PE}$ ?

$\mathrm{D}$ - only detected as the steroid fragment

E - Expected $\mathrm{m} / \mathrm{z}$ ion is not unique and already known to be present

$\mathrm{ND}=$ phosphocholine derivative not detected in serum

This table should not be considered as the list of all of the spiral steroids as there are phosphoester ions that have not been identified. 


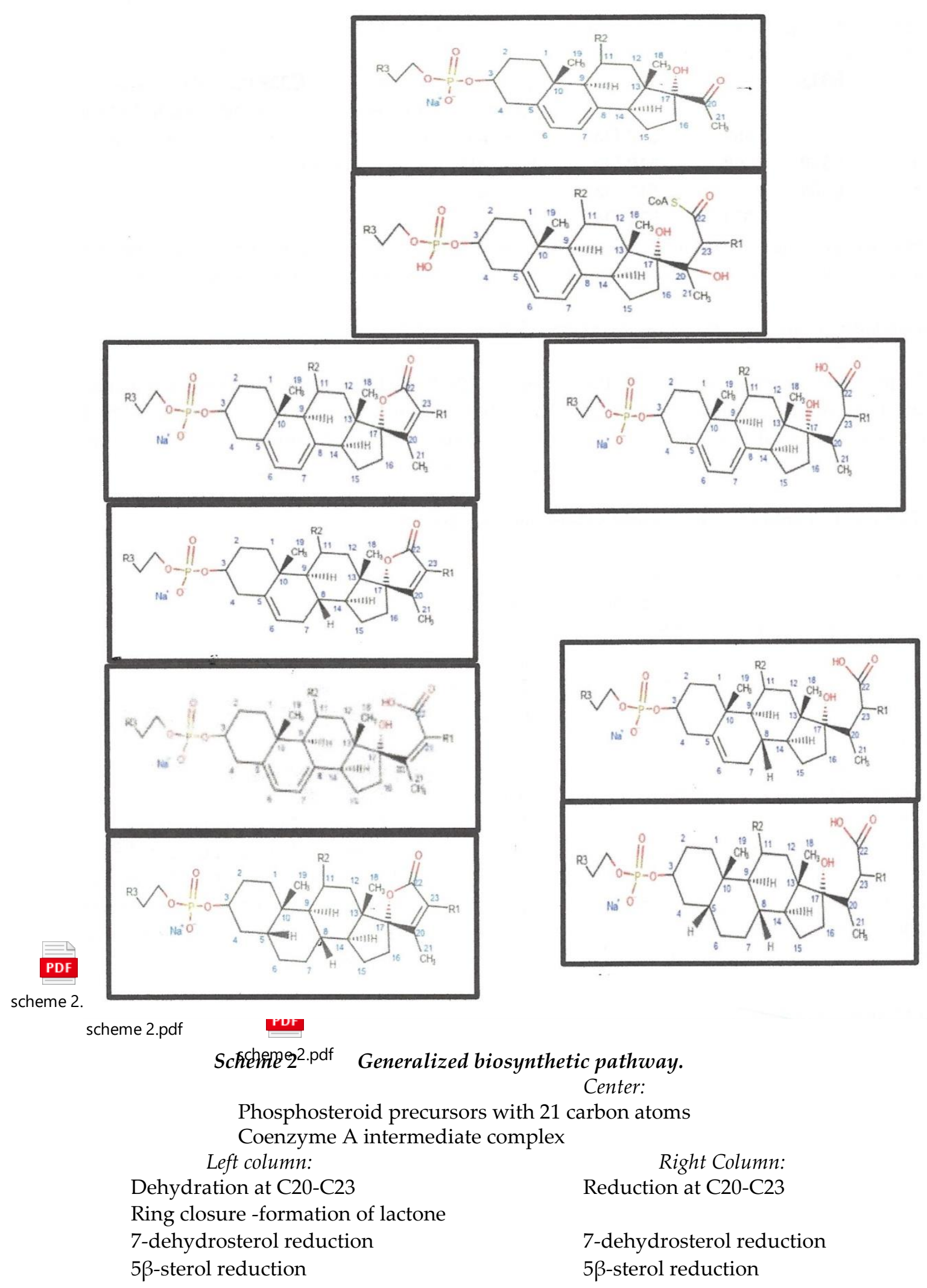

N-Methylation, the conversion of R3 from A to B, can probably occur at more than one step. 
scheme 2.pdf

Table 9: The R Groups on Scheme 2

\begin{tabular}{|c|c|c|c|}
\hline & R1 & R2 & R3 \\
\hline A & $\mathbf{H}$ & $\mathbf{H}$ & $\mathbf{N}^{+} \mathbf{H}_{3}$ \\
\hline B & $\mathrm{CH}_{3}$ & $\mathrm{OH}$ & $\mathbf{N}^{+}\left(\mathrm{CH}_{3}\right)_{3}$ \\
\hline $\mathbf{C}$ & $\mathrm{CO}^{-} \mathrm{CH}_{3}$ & & \\
\hline
\end{tabular}

The table shows the possible range of each of the $\mathrm{R}$ Groups for the structures shown in Scheme 2. For each $\mathrm{R}$ group there is more than one alternative. Additional $\mathrm{R}$ groups are possible but no others have been identified. The three R1 choices are the three most common acyl-Coenzyme A compounds.

\subsubsection{Phosphoesters of high molecular mass}

In addition to the major compounds in adrenals and ovaries, we have noted a few unexpected ions in the spectra from pregnant women. These ions are in the range characteristic of the steroid fragments and have solubility properties of phosphoesters but parent ions were not recognized under the conditions of analysis. When we recognized the role of acyl coenzyme A in the biosynthesis, we have proposed structures that fit the pattern for the two major unexplained peaks (figure 10).
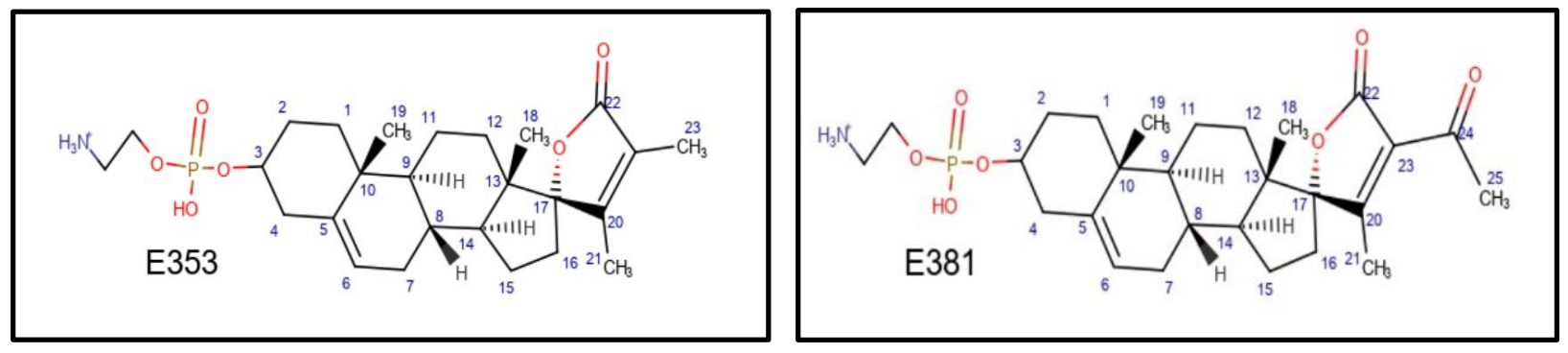

Figure 10. Proposed structures for E353 and E381

\subsubsection{Some final comments of the discovery of the phospho spiral steroid conjugates}

Where possible, in the upper left-hand corner, the codes generated by the instrument are included in the figures. These codes serve to validate the spectra.

\subsection{Application of MS-MS to investigate spiral steroids in samples from individual patients}

To date, investigation of mammalian cardiotonic steroids has been limited because the only method of detection and quantitation has been with antibodies for plant and amphibian toxins. There are no published investigations measuring DLM by LC-MS methodology in serum from individual patients or animals. As described in Section 2.1, there are many compounds that could be detected in various proportions by antibodies to various toxins. The circumstances are now different. We actually isolated $>1$ $\mathrm{mg} / \mathrm{L}$ of C341 and C313 from porcine blood. With this huge endogenous level, we developed a MS based method of quantitating individual Cxxx levels in individual samples. The method was first used with samples from patients with documented enzyme defects. The following sections review the first investigations of specific spiral steroid phosphoesters in serum from patients. 


\subsubsection{Investigation of serum from pediatric patients}

For this investigation, serum samples from 40 pediatric patients were collected in the normal course of patient care [11]. There were 5 groups $(n=6-8)$ and a few samples from children with less common diagnosis. The big groups: were: (1) GH deficiency on therapy, (2) type 1 diabetes on therapy, (3) thyroid disorders on appropriate therapy and (4) patients with 21-hydroxylase deficiency on therapy. Most of samples were from pre-pubertal children. LC-MS analysis of these samples were essentially indistinguishable whether the patients were males or females.
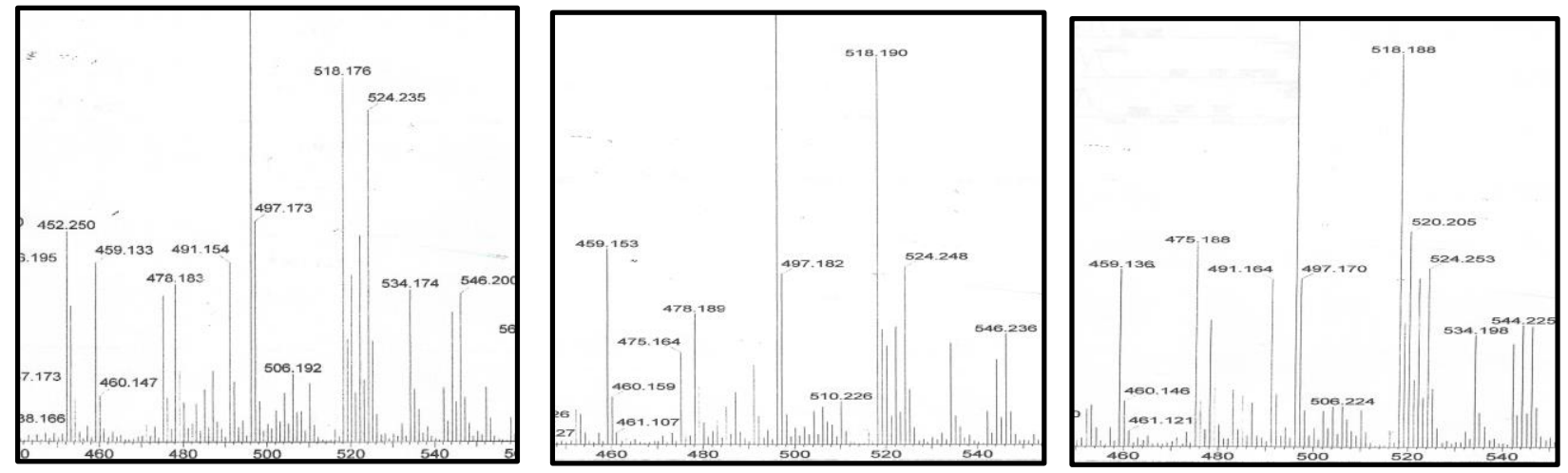

Figure 11. HPLC-MS analysis of serum samples from female pediatric patients. (11)

Left panel: LC-MS spectrum from a 7 year-old female patient with 21-hydroxylase deficiency and premature puberty. The ion peaks at $\mathrm{C} 337(\mathrm{~m} / \mathrm{z}=520 \mathrm{Da}), \mathrm{C} 339(\mathrm{~m} / \mathrm{z}=522 \mathrm{Da})$, and C341 (m/z=524 Da) are the $\mathrm{H}+$ ions. Their presence indicates that biosynthesis does not require 21-hydroxylation. This confirmed the observation that DLM was present in patients with salt-wasting forms of 21- hydroxylase deficiency [26].

Central panel: LC-MS spectrum analysis of a 17 year old female who had not yet started puberty. Note the low levels of the ions at $\mathrm{m} / \mathrm{z}=475 \mathrm{Da}$ and $\mathrm{m} / \mathrm{z}=491 \mathrm{Da}$.

Right panel: LC-MS spectrum from a 19 year old female with polycystic ovarian disorder. The relative amounts of $\mathrm{m} / \mathrm{z}=520 \mathrm{Da}, 522 \mathrm{Da}, \& 524 \mathrm{Da}$ are different indicating, there may a disorder in the production of the spiral lactones, and the levels of both $\mathrm{m} / \mathrm{z}=475 \mathrm{Da}$ and $\mathrm{m} / \mathrm{z}=491 \mathrm{Da}$ are elevated. Thus, there seems to be a disorder in the biosyntheis of the spiral lactones and an excess production of the ions at $\mathrm{m} / \mathrm{z}=475 \mathrm{Da}$ and $\mathrm{m} / \mathrm{z}=491 \mathrm{Da}$. One possible explanation for these ions is they are $\mathrm{Na}+$ and $\mathrm{K}+$ ions of C329 (11 $\beta$-hydroxyl-C313) after loss of trimethylamine.

In all three panels, the central ion at $\mathrm{m} / \mathrm{z}=496 \mathrm{Da}$ is off scale. The $\mathrm{m} / \mathrm{z}=496 \mathrm{Da}$ ion is the $\mathrm{H}+$ parent ion for C313, just like ions at $\mathrm{m} / \mathrm{z}=520 \mathrm{Da}, \mathrm{m} / \mathrm{z}=522 \mathrm{Da}, \mathrm{m} / \mathrm{z}=524 \mathrm{Da}$ correspond to C337, C339, and C341.

\subsubsection{Investigation of serum from patients with 7-dehydrosterol reductase deficiency.}

Using our direct MS assay method, we evaluated spiral lactone biosynthesis in patients with 7dehydrosterol reductase deficiency (Smith-Lemli-Opitz syndrome) [27]. Dr. Porter was able to provide 12 samples, 6 from patients with the syndrome ( 3 males and 3 females) serum from older children with the syndrome and 6 from obligate heterozygotes ( 3 males and 3 females) but was not able to provide samples from the newborn period. The samples were evaluated without knowledge of the diagnosis but it was quite easy to assign the samples to the proper diagnostic category. Representative spectra are shown in Figure 10. Comparison of the spectra indicates that the serum from the affected patients have decreased serum levels of C341, C353, and C381. This suggests that these three compounds require 7-dehydrosterol reductase for their biosynthesis. Conversely, serum from affected patients has significant levels of C313 
(peak at $\mathrm{m} / \mathrm{z}=518 \mathrm{Da}$ ) and $\mathrm{C} 329$ (peak at $\mathrm{m} / \mathrm{z}=475 \mathrm{Da}$ ), indicating that these two compounds probably have a $\Delta 7-8$ alkene element in their structure. The low levels of the steroids, rather than their complete absence, are caused by incomplete blockage of the reductase. During the fetal period, potassium is only provided by maternal plasma $(5 \mathrm{mM} \mathrm{K}+)$ and a hormone that stimulates $\mathrm{K}+$ recovery would be needed. High levels of precursors would be expected to be present [28]. After parturition, the need for a potassium sparing hormone is limited because mother's milk is high in potassium, reducing the need for a $\mathrm{K}+$ sparing hormone. Thus, this pattern is consistent with our index case [8].
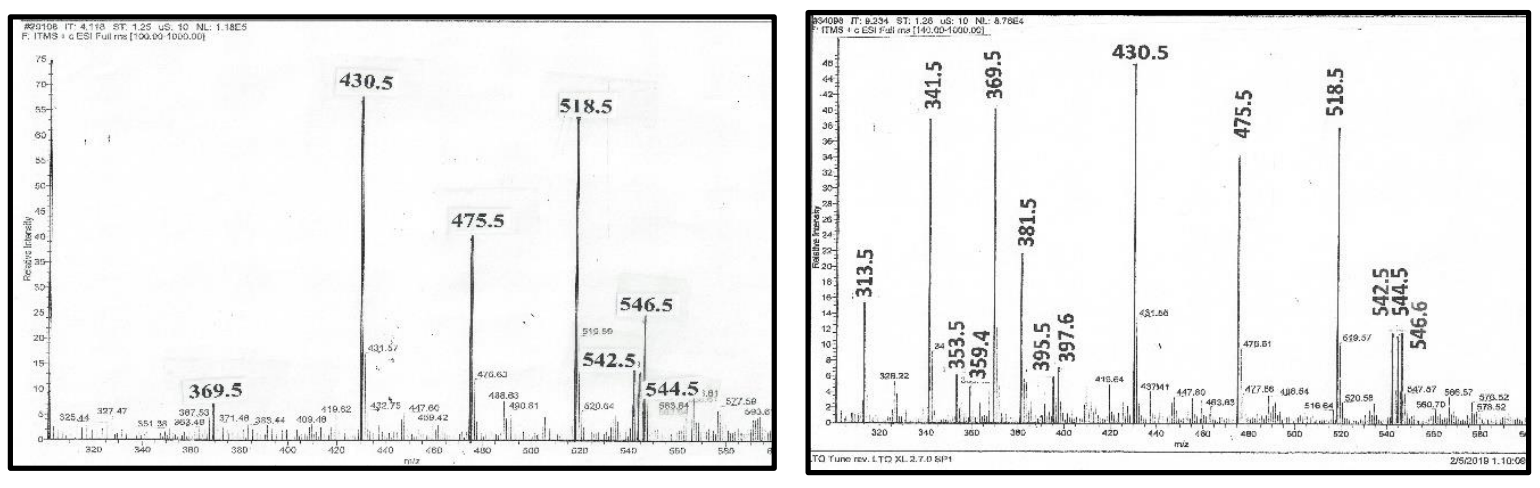

Figure 12. MS Spectrum generated from a patient with 7-dehydrosterol reductase deficiency (left panel) and an obligate heterozygote (right panel). Ion peaks present in the right panel but not present in the left panel identify compounds requiring 7-dehydrosterol reductase for their biosynthesis. With this assay method, most of the PC compounds are present as the Na+ salt. As shown in this figure, for example, C341 as the $\mathrm{H}+$, has $\mathrm{m} / \mathrm{z}=524 \mathrm{Da}$ and as the $\mathrm{Na}+$, has $\mathrm{m} / \mathrm{z}=546 \mathrm{Da}$. Although we have identified most of the major compounds in serum from adults, there are still a few minor ones that remain unidentified, for example the ions at $395 \mathrm{Da}$ and $397 \mathrm{Da}[27]$.

\subsubsection{Investigation of serum from pregnant women}

During pregnancy, pre-eclampsia is the single largest cause of morbidity and mortality. It effects 3 to $5 \%$ of pregnancies [29]. Diagnosis is based on the occurrence of both hypertension and proteinuria after 20 weeks of gestation. There is no FDA approved diagnostic method and no therapy. As gestation proceeds, maternal blood pressure increases. If the blood pressure becomes life threatening, the baby is delivered, immediately. After parturition, there is a long-term 2-4 fold increased risk of poor cardiac function for both the affected infant and the mother. The mother also has a 10-fold increased risk of end stage kidney failure [30]. The underlying biochemistry of the enhanced risks is completely unknown. DLM levels increase during gestation [31] but the pattern is very variable [32]. In animal models, administration of either ouabain or marinobufagenin leads to kidney failure [33]. During the second and third trimester, aldosterone signaling is blocked. Pregnant women get pseudo-hypoaldosteronism (high aldosterone levels, but no mineralocorticoid function) and hypoaldosteronism (low aldosterone levels and no mineralocorticoid function. Thus, there are two different unexplained events during normal pregnancy [34]. During the third trimester, whatever its basis, the absence of mineralocorticoid function causes $\mathrm{Na}+$ wasting and is a key regulatory factor in amniotic fluid production. At birth, cord serum has high levels of DLM and, although aldosterone levels are normal, infants are $\mathrm{Na}+$ wasting and fail to grow until, during the $2^{\text {nd }}$ week post-partum, DLM is no longer present in serum and $\mathrm{Na}+$ wasting ends [28].

It is our theory that these are all related events and dependent on an unidentified spiral steroid hormone [28]. Cardiotonic steroids, detected as assays for DLM, ouabain and marinobufagenin, are all detecting the same hormone. However, no cardiotonic steroid has ever been detected in a vertebrate by 
any technique other than cross-reaction with antibodies specific to one of the plant or amphibian cardiotonic steroids.

For this investigation, we acquired 40 samples from Global Alliance for the Prevention of Prematurity and Stillbirth (GAPPS) [35]. Twenty samples were from women diagnosed with pre-eclampsia and 20 samples were from normotensive pregnant women who were matched for gestational age and sex of the fetus. For analysis, after addition of miltefosine, each sample was extracted with acetonitrile and analyzed as described in the Methods section. Peak intensities were compared to the intensity of the miltefosine peak at $\mathrm{m} / \mathrm{z}=430 \mathrm{Da}$. This ion is the $\mathrm{Na}+$ ion. The low intensity of the ion at $\mathrm{m} / \mathrm{z}=446 \mathrm{Da}$, which would be the corresponding $\mathrm{K}+$, ion confirms the low concentration of $\mathrm{K}+$ ions in the original serum samples. This simplifies the analysis.

Figure 13. Left Panel: MS analysis of a serum extract from a normotensive pregnant woman [35].

Right Panel: MS analysis of a serum extract from a woman with pre-eclampsia.

Note the absence of an ion at $\mathrm{m} / \mathrm{z}=446 \mathrm{Da}$, which would be the $\mathrm{K}+$ of miltefosine

Table 10: Identification of the ions in the serum from pregnant women
Line $\mathrm{m} / \mathrm{z}$
Lactone \# of
R1
R2 Symbol
Alkene Choline DLM

(Da)

C

$\begin{array}{llll}1 & 353 & \text { Yes } & 24 \\ 2 & 381 & \text { Yes } & 25\end{array}$

B

A

P353@

$5 \quad$ NP Unknown

C

A

$5 \quad \mathrm{NP}$

Unknown
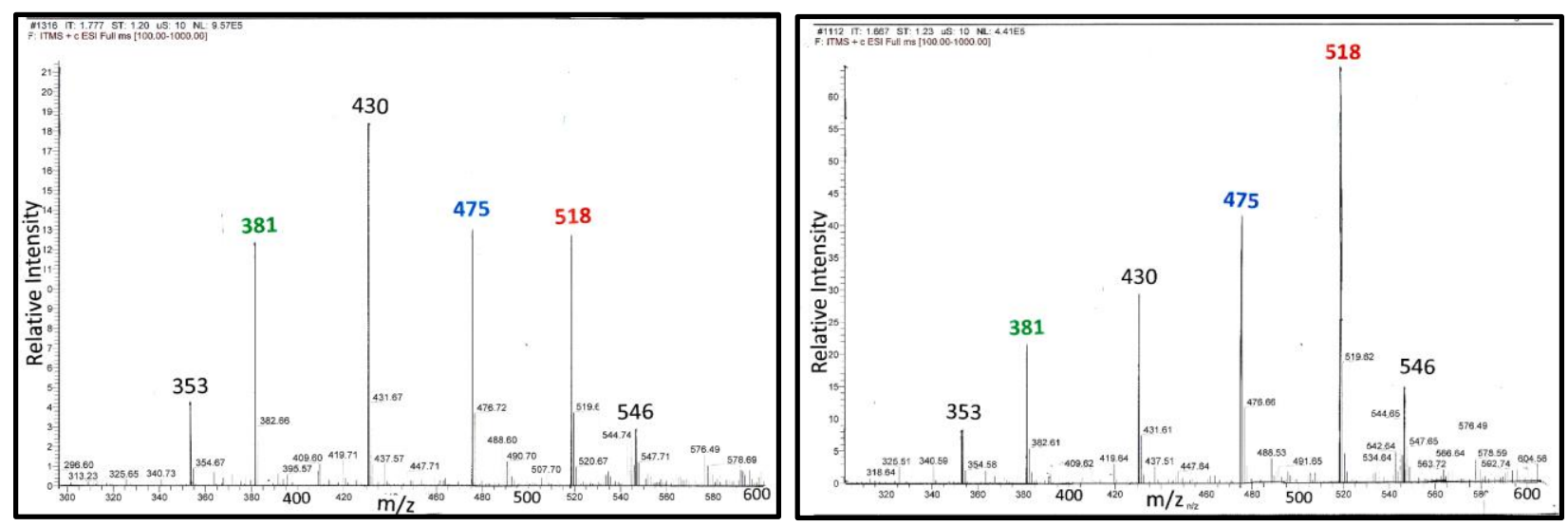

$3430^{*} \quad$ Miltefosine $\mathrm{Na}+$ salt

$\begin{array}{llllllllll}4 & 475^{*} & \text { No } & 21 & \text { NP } & \text { B } & \text { C329 } & 5,7 & \text { Yes } & \text { No } \\ 5 & 518^{*} & \text { No } & 21 & \text { NP } & \text { A } & \text { C313 } & 5.7 & \text { Yes } & \text { No } \\ 6 & 546^{*} & \text { Yes } & 23 & \text { A } & \text { A } & \text { C341 } & \text { none } & \text { Yes } & \text { Yes }\end{array}$

* $\mathrm{Na}+$ ions - corresponding $\mathrm{H}+$ ions and $\mathrm{K}+$ ions are only present as trace components 
@ No ions characteristic of intact steroid esters of either intact phosphoethanolamine or phosphocholine were present. However, in the mass spectrometer, other phosphoethanolamine steroid esters fragment by separation of the steroid fragment. The trial and error analysis for $370 \mathrm{Da}$ (P353) and for $398 \mathrm{Da}$ (P381) only identifies one possible composition. The proposed composition is consistent with a spiral steroid which could be synthesized with the Scheme 2 pathway. We have not identified an appropriate rich source for isolation and have not confirmed that inference.

NP - Not present
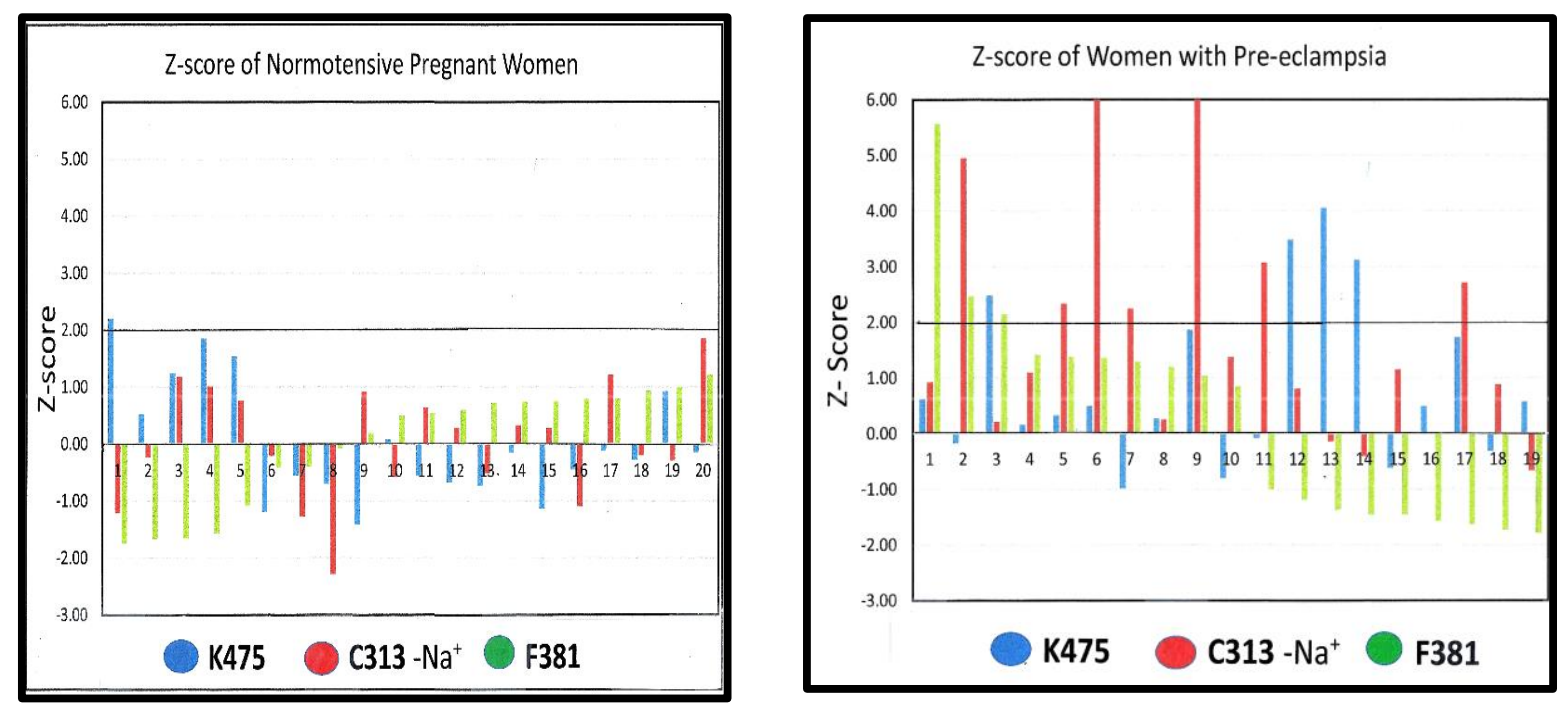

Figure 14. The relative intensities of the different ions present in serum from pregnant women [35] Left Panel: analysis of serum samples from normotensive pregnant women Right Panel: analysis of serum samples from women with pre-eclampsia.

Method of evaluation: For each spectrum, the intensity of each ion was compared to the intensity of the ion generated by miltefosine. The mean and standard deviation of ions from the normotensive women were calculated and used to generate Z-scores for each of the 40 samples. The Z-scores for each sample were graphed as a cluster.

In the samples from the normotensive women, only one sample had a single value with a Z-score $>2$. The results evaluating the samples from the women with pre-eclampsia are shown on the right. Twelve of the samples had at least one sample with a z-score over 2. That distribution is statistically significant at the o.o5 level. Thus, there seems to be two different diseases. One disease associated with normal levels of PC steroids and a second disease with high levels of PC steroids. However, high levels of C313 and K475 could not account for the high DLM levels in cord serum because neither compound is detected by digoxin specific antibodies [36].

Originally, we thought that the K475 compound was derived from the K+ ion of C313 (m/z=534 Da) after the loss of its trimethylamine fragment. However, GAPPS advised us that no K+ containing reagents were used to prepare any serum samples. Secondly, a magical process would be needed to select only one phosphocholine ion for binding to $\mathrm{K}+$ and not any other, such as miltefosine. We now think that the K475 compound derives from C329 - the 11-hydroxy sister of C313. We now have three sub-groups of patients with pre-eclampsia: Type 1 with high levels of C313, Type 2 with high levels of C329 and Type 3 with low levels of both C313 and C329.

The gestational stage of the women who provided serum for our investigation ranged from 24 to 27 weeks. Symptoms of pre-eclampsia continue to develop as pregnancy progresses. Further changes in the spiral steroids, both qualitative and quantitative, would be expected during the $3^{\text {rd }}$ trimester.

Questions: 
- Which sub-group is at risk of developing life-threatening hypertension?

- Which sub-group is at increased risk of developing renal or cardiac disorders?

- Does persistence after parturition explain the long term increased risk of renal failure?

- Are there differences in the spiral steroids in the fetal circulation?

The ratio of fetal blood volume to maternal blood volume is about 50:1. It is quite possible for the fetus to use C313 or C329 for local synthesis of a spiral lactone for an unknown function. Perhaps C329 binds to the mineralocorticoid receptor and blocks aldosterone signaling. Both compounds have $11 \beta$ hydroxyl groups. Could that be part of the nuclear receptor binding site?

\subsubsection{Is there a role for spiral steroids in Post-Trauma Stress Disorder (PTSD)?}

PTSD has been a major interest at the VA Medical Center. Diagnosis is routinely made on the basis of DSM-5 criteria. Once the diagnosis has been established, endocrinologists have noted symptoms that seem to be common in affected patients, typically: (a) inadequate GH secretion, (b) lack of REM sleep, (c) inadequate production of cortisol, despite over secretion of ACTH [37] and (d) hypertension [38]. The steps (a) - (b) - (c) - (x) - (y) - (d) seem to form a cascade but the connection between (c) and (d) has not been recognized. The observation that individuals who experience lack of REM sleep are GH deficient is well known. Less well known is that GH deficient patients secrete a higher proportion of corticosterone to cortisol, then do individuals with adequate GH secretion [39]. Although cortisol is about 4 times more potent than corticosterone as a glucocorticoid, corticosterone is more than ten times more potent than cortisol as mineralocorticoid. This is step (x). Over production of corticosterone causes sodium retention. Step (y) is synthesis of an endogenous potassium sparing hormone. This restores the electrolyte balance but causes hypertension. The recognition of $(x)$ and $(y)$ completes the endocrine cascade. A new approach to therapy for these patients could be to interfere with the endocrine cascade. In summary, the new understanding of the cardiotonic steroids, particularly the function of Ionotropin (C341) as a potassium sparing hormone, suggests that interfering with the endocrine cascade might provide a new therapeutic approach to PTSD [40].

\section{Discussion}

In summary, there are at least 3 classes of spiral lactones - C341 (23 carbon atoms), C353 (24 carbon atoms) and C383 (25 carbon atoms). Based on the various compounds we have characterized, starting with precursors with 21 carbon atoms, the additional carbons are added by condensation of an acyl coenzyme A derivative. These match with the three most common acyl coenzyme A derivatives, which are acetyl, propyl, and acetoacetyl. There are two 21 carbon precursors, C313 and C329, C329 has an extra hydroxy group that might be at carbon 11 . These are both present in serum but the $11 \beta$-hydroxy could also be added later. There are at least 4 members of each class, for example - C337, C339, C341 and C363. There may also be matching compounds with 11 $\beta$-hydroxyl groups.

Based on the isolation of the Exxx compounds, the Cxxx compounds are not synthesized by transfer of phosphocholine directly from other phosphatidyl lipids. The steroidogenic tissues (specifically the adrenal and gonads) all have high concentrations of Exxx compounds but the corresponding serum concentration is minimal. Methylation of norepinephrine to epinephrine is regulated by ACTH and cortisol. These could all be targets for future development of therapeutic agents and for a better understanding of many disease processes.

The observation that there are multiple classes of spiral lactones explains some of the conflicting observations about cardiotonic steroids. It also suggests that investigators should not rely on a single antibody to evaluate the concentration of the active material. Finally, the recognition of both PC and PE spiral steroids points to a new site for regulation. Although most of the steroid hormones function via nuclear receptors, that is clearly not the case for all of the phosphoester compounds. C341 is present in 
serum at a concentration of $1 \mathrm{mg} / \mathrm{L}$. If it functioned molecule for molecule with a nuclear receptor, it would require $2000 \mathrm{mg}$ of receptors for each liter of blood. Hence, the steroid phosphoesters must function by a different mechanism. One mechanism that we already have recognized is via the NaK-ATPase. However, that is only half the story because the apparent half-life is too long to regulate the changes that must take place when we eat or drink. Although the compounds we have isolated and detected in serum are present in high concentration, there may be others that function by classical receptor mechanisms. Because of the relative small size of gonads and the fetus, when compared to an adult weighing $50 \mathrm{~kg}$, this is particularly likely during reproductive processes including fetal development.

\section{Methods:}

Extraction: Tissue or blood was homogenized with a mixture of acetonitrile/water, to yield a final ratio of about 4:1. In batches, the extract was applied to a preparative amino carbohydrate HPLC column (22x500 mm) from Alltech (Chicago). Aliquots of each fraction were tested with a saturated silver nitrate reagent. When the test was positive (white precipitate forms), the application of the crude extract was ended and the column eluted with a 40-minute gradient of water/acetonitrile (20/80) to water/acetonitrile (60/40). Individual fractions were analyzed by LC-MS. Fractions containing phosphocholine esters were combined and concentrated under vacuum. Three passes were necessary three to get a single peak. The final step was isocratic elution at water/acetonitrile (18/82). As an example, starting with 10 liters of porcine blood, the final sample weighed about $8 \mathrm{mg}$. If the isolation process recovered $50 \%$ of the amount in porcine blood, then the original serum concentration was about $1.6 \mathrm{mg} / \mathrm{L}(160 \mu \mathrm{g} / \mathrm{dL})$ or $3 \mu \mathrm{M}$.

The original analytical LC-MS procedure was as follows: [a] $0.1 \mathrm{ml}$ of serum from a patient sample was pipetted into a $12 \times 75 \mathrm{~mm}$ tube, [b] to each tube $0.4 \mathrm{~mL}$ of acetonitrile was added, [c] each tube was vortexed, [d] the sample was filtered through a syringe filter, and [e] subject to LC-MS after transfer to an injection vial (MS was Waters, APCI in the positive ion mode). Accelerating voltages were adjusted to minimize fragmentation. This method was used for the pediatric samples and for the original purification of the steroid phosphocholine esters.

NMR spectra were obtained by Acorn Laboratories (Livermore, CA).

Trial and error analysis table of possible chemical formulas containing only carbon, oxygen and hydrogen atoms. Each line shows a possible combination of carbon and oxygen atoms necessary to generate a specific mass. The $\mathrm{C} \& \mathrm{O}$ column shows how many Da would be provided by the specific combination of carbon and oxygen atoms on that line. H-Req shows the required number of hydrogen atoms necessary to complete the molecule based on the difference between $\mathrm{C} \& \mathrm{O}$ and 330, the indicated mass of the steroid fragment. Delta is the sum of rings, double bonds and other features that provide the required number of hydrogens. Two hydrogens would be needed for each Delta. Because a steroid has 4 rings, Delta can't be less than 4 nor more than 12 without an aromatic A ring. The line in RED in each table shows the only combination that can make a steroid-like molecule. Multiple isomers are possible but only one chemical formula is possible. The analytical method won't always provide a unique composition, but it does for C341 and most of the other phosphoesters.

Analytical method for analysis of individual serum samples, $10 \mu \mathrm{l}$ of a $0.2 \mathrm{mg} / \mathrm{ml}$ solution of miltefosine (Cayman Chemical, Ann Arbor, MI) was added to $0.2 \mathrm{ml}$ of serum. The serum was extracted with $0.8 \mathrm{ml}$ of acetonitrile, centrifuged and filtered with Whatman Syringe filters with $0.2 \mu \mathrm{m}$ pore size. The filtrate was analyzed by direct injection into the electrospray source of a LTQ-XL quadrupole ion trap mass spectrometer (MS) (Thermo Scientific, San Jose, CA). Flow rate was $10 \mu \mathrm{l} / \mathrm{min}$. The capillary temperature was 275 C. Spray voltage was 4.4 volts. Ten mass spectra scans were collected and averaged for quantification. Each sample was also analyzed when 60 volts was applied at the source to fragment the molecules. Representative peaks were selected for MS/MS to identify parent/fragment ion relationships. For selection of the parent ions for collision- and induced dissociation, the isolation width was set to 2 . All spectra were generated in the positive ion mode. 


\section{Conclusions:}

5.1 What did we Discover

- Spiral steroids with 23 carbon atoms

- Phosphoester conjugates

- Precursors in bovine adrenal cortex and ovarian extracts

- Related spiral steroids with 24 and 25 carbon atoms

- A new class of $11 \beta$-hydroxy steroids

- Serum concentrations sufficient for detection by mass spectroscopy with less than $1 \mathrm{ml}$ of serum

- Evaluation of serum concentration may be useful for disease diagnosis

- Many new targets for transformational development

\subsection{Overview}

Although it is unlikely that every proposed structure is correct, certain basic elements should not be ignored:

- Ionotropin, C341 is a an endogenous DLM. Based on its detection by digoxin specific assays, it is a digoxin-like material. It was isolated from human, bovine, and porcine serum. LC-MS analysis shows the absence of major contamination. There is no evidence for a glycoside component, as is present in the plant cardiotonic glycosides. Isolated Ionotropin inhibits NaK-ATPase just like other cardiotonic steroids. In brief, Ionotropin fits the definition of a cardiotonic steroid.

- Ionotropin is a phosphocholine ester. It fragments in two ways: loss of trimethylamine and loss of phosphocholine. The ${ }^{31} \mathrm{P}-\mathrm{NMR}$ spectrum shows the presences of a phosphate. A synthetic phosphocholine ester, DHEA-PC, fragments in the same pattern and also has a similar ${ }^{31} \mathrm{P}-\mathrm{NMR}$.

- Based on the a-phosphocholine, the mass of the steroid portion of Ionotropin is $358 \mathrm{Da}$. This is equivalent to calculating the mass of digoxigenin by subtracting the mass of the a-glycone from the mass of digoxin.

- The trial and error method of determining the chemical composition produces only a single result. This need not be the general case, but it is here. This doesn't mean that that there couldn't be isomers but that wouldn't change the points above. Ionotropin would still be an endogenous mammalian cardiotonic steroid. Other scientists are welcome to propose an alternate structure consistent with the overall pattern of steroid phosphocholine esters that is described here.

\subsection{The Significance}

Our efforts have concentrated on the isolation, characterization and biosynthesis of these compounds. Nicholls has proposed that the plant and amphibian cardiotonic steroids are not truly endogenous [41]. This paper reviews the discovery process for the spiral steroid phosphoesters. The data satisfies Nicholls guidelines for an endogenous cardiotonic steroid: (1) isolation and structural identification, (2) precursors and biosynthesis, and (3) correlation with at least one disease process. The hormonal function and regulation of these novel hormones presents the possibility of improved understanding of many endocrine processes. This discovery puts the role of plant and amphibian toxins in a new light. Investigators have now to identify which one is associated with a particular function and/or disorder. 


\section{Declarations}

\section{Ethics approval and consent to participate:}

Serum from Pediatric patients was collected by Dr. Alicia Romano. Dr. Romano had IRB approval from her Hospital. Coded samples were received in the laboratory without personal identification. After the samples were evaluated, the code was broken and tested for clinical correlation.

Serum samples from patients with 7-dehydrosterol reductase deficiency were collected by Dr. Forbes Porter of the NICHD and stored for future investigation. Coded samples were received in the laboratory without personal identification.

Samples from pregnant women were collected as part of database collection program and stored by the Global Alliance for the Prevention of Prematurity and Stillbirth (GAPPS). Searching their database for the key phrase "pre-eclampsia/eclampsia," we selected 20 case samples and were then provided with 20 controls for a total of 40 specimens. Patient demographics were categorized and provided by GAPPS, but for the purposes of our study, only the variables pre-eclampsia/eclampsia were utilized as our sample size was to small to make any inferences regarding any other patient demographic variable. Samples were coded by GAPPS without any patient identifiers and analyzed without knowledge of the disease status. The disease status code was broken only after results were shared.

\section{Authors' contributions:}

FC initiated the current investigation, generated the laboratory data, and wrote the manuscript.

\section{Funding Statement:}

This research did not receive any specific grant from funding agencies in the public, commercial or not-forprofit sectors. Dr. Ron Bochner personally funded the pilot study of women with pre-eclampsia. The Smith-LemliOpitz Foundation funded the investigation of the patients with SLO syndrome. AMUR Research Corp funded most of original investigations. Kerix funded the purification of the compounds with 23 carbon atoms and the investigation of the chemical formulas. More recently, this work was partially supported by the Research Service of the United States Department of Veterans Affairs, by Dr. Gary Jarvis, VA Medical Center, San Francisco, CA and by Dr. Constance John, VA Medical Center, San Francisco, CA. Current support is from IOMA LLC.

\section{Acknowledgements:}

I specifically wish to acknowledge my two very special colleagues - Dr. H. Leon Bradlow and Dr. Sandra Blethen. I acknowledge Dr. Forbes Porter and Dr. Christopher Wassif of the NICHD for providing serum samples from patients and obligate heterozygotes and Dr. Alicia Romano for providing samples collected in the normal course of her patient care. This work was partially supported by the Research Service of the United States Department of Veterans Affairs, by Dr. Gary Jarvis, VA Medical Center, San Francisco, CA and by Dr. Constance John, VA Medical Center, San Francisco, CA. Dr. John specifically encouraged me and made laboratory space and equipment available. My two laboratory chiefs were Michael Davis and Lori Pierce-Cohen.

Marvin Applets were used for drawing, displaying and characterizing chemical structures and reactions, Product Version 20.1 ChemAxon (https://www. Chemaxon.com).

Conflicts of Interest: Author declares no conflict of interest.

$\begin{array}{llll}\text { Abbreviations } & & \\ \text { MS } & \text { Mass spectroscopy } & \text { MS-MS } & \text { Tandem mass spectroscopy } \\ \text { NMR } & \text { Nuclear Magnetic Resonance } & \text { CS } & \text { Cardiotonic Steroids } \\ \text { PC } & \text { Phosphocholine } & \text { PE } & \text { Phosphoethanolamine } \\ \text { DLM } & \text { Digoxin-like Materials } & & \end{array}$




\section{References:}

1. Withering W. An account of the foxglove and some of its medical uses: with practical remarks on dropsy and other diseases. 1785: G. G. J. \& J. Robinson. London.

2. Szent-Gyorgyi A. Chemical physiology of contraction in body and heart muscle. Academic Press. New York, NY. (As cited in Labella FS. Endogenous digitalis-like factors: Introductory Remarks. LaBella FS. Fed Proc.1985. 44: 2780-2781.

3. Walsh $\mathrm{P}$, Crawford F, Hawker CD. Measurement of digoxin by radioimmunoassay. Annals of Clinical and Laboratory Science. 1977. 7: 79-87.

4. Hamlyn J, Blaustein M, Bova S, DuCharme D, Harris D, Mandel F, Mathews W, Ludens J. Identification and characterization of a ouabain-like compound from human plasma. Proc Natl Acad Sci. 88: 6259-6263 (1991).

5. Bucalew V. Endogenous digitalis -like factors: an overview of the history. Frontiers in Endocrinology. 2015. 6: Article 49: 1-9.

6. Baecher S, Kroiss M, Fassnacht M, Vogeser M. No endogenous ouabain is detectable in human plasma by ultrasensitive UPLC-MS/MS. Clin Chim Acta, 431: 87-9 (2014).

7. Smith DW, Lemli L, Opitz M. A newly recognized syndrome of multiple congenital anomalies. J Pediatrics. 64: 210-217 (1964). Pubmed/14119520.

8. Chasalow F, Blethen S, Taysi K. Possible abnormalities of steroid secretion in children with Smith-Lemli-Opitz syndrome and their parents. Steroids. 1985. 46: 827-843.

9. Bradlow H, Fleisher M, Breed C, Chasalow F. Biochemical classification of patients with gross cystic breast disease. N Y Acad Sci. 1990. 586:12-16.

10. Chasalow FI, Bradlow HL. Digoxin-like materials in human breast cyst fluids. Ann N Y Acad Sci. 1990; 586:10716. doi: 10.1111/j.1749-6632.1990.tb17797.x. PubMed PMID: 2162647.

11. Chasalow F, Pierce-Cohen L. Ionotropin is the mammalian digoxin-like material (DLM). It is a phosphocholine ester of a steroid with 23 carbon atoms. Steroids. In press (2018). DOI.org/10.1016/j.steroids.2018.03.001

12. Shackleton C, Roitman, E, Guo LW, Wilson WK, Porter FD. Identification of 7(8) and 8(9) unsaturated adrenal steroid metabolites produced by patients with 7-dehydrosterol-delta-7-reductase deficiency (Smith-Lemli-Opitz Syndrome). J Steroid Biochem Mol Biol. 82: 225-32 (2002). Pubmed/12477489

13. Synthesis of DHEA-S Chasalow FI. Phospholipid drug derivatives. US Patent 6,127,349 (2000).

14. Burstein S, Gut M. Biosynthesis of pregnenolone. Recent Prog Horm Res. 1971;27:303-49. doi: 10.1016/b978-0-12-571127-2.50032-8.PMID: 4946132

15. Slominski A, Ziawiony J, Wortsman J, Semak I, Stewart J, Pisarchik A, Steatman T, Marcos J, Dunbar C, Tuckey R. A novel pathway for sequential transformation of 7-dehydro-cholesterol and expression of the P450 SCC system in mammalian skin. Eur J Biochem. 271: 4178-4188 (2004).

16. Slominski A, Zmijewski M, Semak I, Sweatman T, Janjetovic Z, Li W, Zjawiony J. Sequential metabolism of 7dehydrocholesterol to steroid 5,7-dienes in adrenal glands and its biological implication in the skin. PLoS ONE 4(2): e4309 (2008). Doi: 10.1371/journal.pone.0004309.

17. Wassif C, Maslen C, Kachilele S, Lin D, Linck L, Connor W, Steiner R, Porter F. Mutations in the Human Sterol $\mathrm{A}^{7}+$ Reductase Gene at 11q12-13 Cause Smith-Lemli-Opitz Syndrome. Am J Hum Gen. (1988) 63: P55-62. https://DOI.org/10.1086/301936.

18. Gonzales, E.t Matarazzo, L, Franchi-Abella, S. et al. Cholic acid for primary bile acid synthesis defects: a lifesaving therapy allowing a favorable outcome in adulthood. Orphanet J Rare Dis(2018) 13, 190. https://doi.org/10.1186/s13023-018-0920-5

19. Chasalow FI. A New Concept: Ionotropin might be a Factor in Mobilization for [a] the Fight or Flight Response and [b Child Birth. Pediatrics (E-cronicon) 7(9) 909-918 (2018). doi: 10.31080/ecpe.2018.07.00341

20. Gibellini F, Smith T. The Kennedy Pathway - De Novo Synthesis of phosphatidyl ethanolamine and Phosphatidylcholine. IUBMB Life, 2010. 62 (6): 414-428 (2010). DOI: 10 .1002/iub.337 
21. Bleijerveld O, Browers J. Vaandrager A, Helms J, Houweling M. The CDP-ethanolamine pathway and phosphatidylserine decarboxylase generate different phosphatidylethanolamine molecular species. J Biol Cdoi:hem 282: 28362-28372 (2007). DOI10.1074/jbc.M703786200.

22. Bobenchik A, Augagneur Y, Hao B, Hoch J, Mamoun C. Phosphoethanolamine methyltransferases in phosphocholine biosynthesis: functions and potential for antiparasite therapy. FEMS Microbiol Rev, (2011) 35: 609-619. doi: 10.1111/j.1574-6976.2011.00267.x

23. https://www.thoughtco.com/why-rotten-eggs-float-4116957

24. Chasalow F. Spiral Phosphocholine Steroids and DLM in Chicken Eggs (Gallus gallus domesticus). EC Paediatrics 8.10 (2019): 01-12.

25. Hamlyn J, Laredo J, Shah J, Lu Z, Hamilton B. 11-hydroxylation in the Biosynthesis of Endogenous Ouabain: Multiple Implications. Ann NY Acad Sci (2003) 986: 685-93. Doi : 10.1111/j.1749-6632.2003. tb07283.x

26. Blethen SL, Chasalow FI. Characterization of a digoxin-like material in salt-wasting congenital adrenal hyperplasia (CAH). 3rd Joint Meeting European Society for Pediatric Endocrinology/ Lawson Wilkins Pediatric Endocrine Society. 1989; Jerusalem, Israel.

27. Chasalow F, Blethen S. Steroid Metabolic Consequences of 7-Dehydrosterol Reductase Deficiency (SLO). EC Paediatrics (2020) 9: 60.69.

28. Chasalow F. A new concept for the regulation of electrolytes in pregnancy: The role of Ionotropin, the endogenous potassium sparing hormone. EC-Pediatrics. 7 (6): 527-532 (2018). doi: 10.31080/ecpe.2018.07.00267

29. Ananthe CV, Keyes KM, Warner RJ. Pre-eclampsia rates in United States 1980 to 2010: age-period-cohort analysis. BMJ 347: f6564. (2013). doi: https://doi.org/10.1136/bmj.f6564

30. http://www.preeclampsia.org/health-information/about-preeclampsia

31. Graves S. The Possible role of Digitalislike Factors in Pregnancy-Induced Hypertension. Hypertension 10 \{suppl I\}: 1987. I-84- I-86.

32. Buckalew V. Role of endogenous digitalis-like factors in the clinical manifestations of severe pre-eclampsia: a systematic review. Clin Sci 132: 1215-1242 (2018). doi: 10.1042/cs20171499

33. Fedorva L, Raju V, El-Okdi N, Shidyak A, Kennedy D, Vetteth S, Giovannucci D, Bagrov A, Fedorva O, Shapiro J, Malhotra D. The cardiotonic steroid hormone marinobufagenin induces renal fibrosis: implication of epithelialto-mesenchymal transition. Am J Physiol Renal Physiol (2009) 296: F922-934. Doi:10.1152/ajprenal.90605.2008

34. Bizzarri C, Pedicelli S, Cappa M, Cianfarani S. Water Balance and 'Salt Wasting' in the First Year of Life: The Role of Aldosterone-Signaling Defects. Horm Res Paediatr. 86: 143-153 (2016). DOI:10.1159/000449057.

35. Chasalow F, John C, Bochner R. Spiral steroids as potential markers for pre-eclampsia: a pilot study. Steroids. 2019 Nov;151:108466. doi: 10.1016/j.steroids.2019.108466. Epub 2019 Jul 26. PubMed PMID: 31351941

36. Chasalow F, Blethen S. Characterization of digoxin-like material in human cord serum. Ann N Y Acad Sci. 591: 212-21 (1990). PMID: 2142872.

37. Yehuda R. Biology of Posttraumatic Stress Disorder. J Clin Psychiatry (2001): 62 (suppl 17).

38. Kibler J, Joshi k, Ma M. Hypertension in Relationship to Posttraumatic Stress Disorder and Depression in the US National Comorbidity Survey. Behavioral Medicine. 34:125-131 (2009).

39. Chasalow F, Blethen SL. Modulation of Glucocorticoid Secretion by Growth Hormone. Pediatric Research. (1985) 19:823-827.

40. Chasalow F, Blethen S. New concept: Endocrine Dysfunction in Post-Traumatic Stress Disorder (PTSD): The role of Ionotropin. EC Paediatrics (2019) 8 (10): 1104-1110. Doi: 10.31080/ecpe.2019.08.00560

41. Lewis L, Yandle T, Hilton P, Jensen B, Begg E, Nicholls G. Endogenous Ouabain Is Not Ouabain. Hypertension (2014) 61: 680-683. DOI: 10.1161/HYPERTENSIONAHA.114.03919 